\title{
On the Singular Set of Harmonic Maps into DM-Complexes
}

\author{
Georgios Daskalopoulos ${ }^{1}$ \\ Brown University \\ daskal@math. brown.edu \\ Chikako Mese ${ }^{2}$ \\ Johns Hopkins University \\ cmese@math. jhu.edu
}

\begin{abstract}
We prove that the singular set of a harmonic map from a smooth Riemannian domain to an NPC Riemannian DM-complex is of Hausdorff codimension at least two. This generalizes results of GromovSchoen on harmonic maps into F-connected complexes and includes many interesting target spaces, for example hyperbolic buildings, not covered by Gromov-Schoen. We also explore other interesting properties of harmonic maps to DM-complexes like monotonicity formulas and an order gap theorem for approximately harmonic maps.
\end{abstract}

\section{Introduction}

Harmonic map theory from Riemannian domains to singular spaces originate with the work of Gromov-Schoen [GS] and was subsequently extended in $[\mathrm{KS} 1][\mathrm{KS} 2]$ and $[\mathrm{J}]$. The motivating question comes from the study of superrigidity of group representations. Let $\Gamma$ be a fundamental group of a manifold $M$ acting on a metric space $Y$ by a representation $\rho: \Gamma \rightarrow \operatorname{Isom}(Y)$. Suppose that associated with the action $\rho$, there is an equivariant harmonic map $\tilde{u}: \tilde{M} \rightarrow Y$ where $\tilde{M}$ is the universal cover of $M$. Under appropriate curvature assumptions on the domain and the target spaces one would like to show that the map $\tilde{u}$ is totally geodesic or even constant, thus implying

\footnotetext{
${ }^{1}$ supported by research grant NSF DMS-0604930

${ }^{2}$ supported by research grant NSF DMS-0706933
} 
the rigidity of the representation $\rho$. This is the famous Bochner method and in the case when $Y$ is a smooth manifold it has been extensively used by many authors (cf. for example, [Si], [C], [JY] and [MSiY] among others). Recall, that the Bochner formula is a differential equation involving higher derivatives of the map and relies on the smooth structure of the Riemannian manifolds involved. Therefore, in order to utilize it in the singular setting the key is to show that harmonic maps into singular spaces are regular enough on a big open set.

In the seminal work of Gromov and Schoen [GS], it is shown that this is in fact the case when the target space is an $F$-connected simplicial complex. Roughly speaking, an F-connected complex is a $k$-dimensional Euclidean complex where any two adjacent simplices lie on a flat, i.e. an image of the Euclidean space $\mathbf{R}^{k}$ isometrically embedded in the complex. Examples of F-connected complexes are Euclidean buildings. In doing so, Gromov and Schoen showed that rank 1 superrigidity extends to the non-Archimedean case generalizing the Archimedean superrigidity result of Corlette [C].

More precisely, the main technical result of [GS] is to show that a harmonic map $u$ from a smooth Riemannian domain $\Omega$ to a $k$-dimensional Fconnected complex $Y$ locally maps into a Euclidean space outside a set of codimension at least 2 , or in other words that the singular set $\mathcal{S}(u)$ of $u$ is at least of Hausdorff codimension 2. This is enough to justify the Bochner formula. To investigate the singular points, they show the existence of the order function associated with a harmonic map. For example, for a harmonic function $u: \Omega \rightarrow \mathbf{R}$, the value of the order function $\operatorname{Ord}(x)$ is the order with which $u$ attains its value $u(x)$ at $x$, or alternatively, it is the degree of the dominant homogeneous harmonic polynomial which approximates $u-u(x)$ near $x$.

In this paper, we extend the result of [GS] to NPC (non-positively curved) complexes that are not necessarily Euclidean. Such complexes can have arbitrary Riemannian metrices but we assume that they are DM-connected in the sense that any two adjacent simplices lie in a DM, an image of a differentiable manifold isometrically embedded in $Y$. Examples of such complexes are of course Euclidean and hyperbolic buildings. The main theorem of the paper can be stated as follows

Theorem 1 (Main Theorem) Let $\Omega$ be an n-dimensional Riemannian domain, $Y$ a k-dimensional NPC DM-complex and $u: \Omega \rightarrow Y$ a harmonic map. 
Then the singular set $\mathcal{S}(u)$ of $u$ has Hausdorff co-dimension 2 in $\Omega$; i.e.

$$
\operatorname{dim}_{\mathcal{H}}(\mathcal{S}(u)) \leq n-2 .
$$

A harmonic map $u: \Omega \rightarrow Y$ into a $k$-dimensional DM-complex can be written locally near a singular point $x \in \mathcal{S}(u)$ as $u=(V, v)$ where $V$ is the non-singular component map that maps into a Euclidean space $\mathbf{R}^{j}$ and $v$ is the singular component map that maps into a lower dimensional complex $Y_{2}^{k-j}$. We partition $\mathcal{S}(u)$ as $\bigcup \mathcal{S}_{j}(u)$ where $j$ indicates the dimension of the target space $\mathbf{R}^{j}$ of $V$. When the target space $Y$ is an F-connected complex, $u$ maps into the product of $\mathbf{R}^{j}$ and $Y_{2}^{k-j}$ and both components $V$ and $v$ are harmonic maps. Therefore, the analysis of the singular set of $u$ can be inductively reduced to the study of the singular set of $v$ which maps into a lower dimensional complex. This is in fact how it is argued in [GS]. In the case when the target space is a general DM-complex, $u$ locally maps into the twisted product of $\mathbf{R}^{j}$ and $Y_{2}^{k-j}$ which we denote by $\left(\mathbf{R}^{j} \times Y_{2}^{k-j}, G\right)$. The maps $V$ and $v$ are thus only approximately harmonic. More significantly, the map $v$ is the non-dominant term of $u=(V, v)$ and this presents the major technical difficulty of the paper. In analyzing the singular set of $v$, we prove a general monotonicity formula to deduce the existence of the order function and the order gap theorem for the approximate case. Here we summarize our results:

Theorem 2 (The Order of the Singular Component) Let $\Omega$ be an $n$ dimensional Riemannian domain, $Y$ a $k$-dimensional NPC DM-complex, $u$ : $\Omega \rightarrow Y$ a harmonic map and $j=0, \ldots, k_{0}:=\min \{n, k\}$. If $x_{0} \in \mathcal{S}_{j}(u)$ and $u=(V, v): \Omega \rightarrow\left(\mathbf{R}^{j} \times Y^{k-j}, G\right)$ near $x_{0}$, then

$$
\operatorname{Ord}^{v}\left(x_{0}\right)=\lim _{\sigma \rightarrow 0} \operatorname{Ord}^{v}\left(x_{0}, \sigma\right)=\lim _{\sigma \rightarrow 0} \frac{\sigma E_{x_{0}}^{v}(\sigma)}{I_{x_{0}}^{v}(\sigma)}
$$

exists.

Theorem 3 (The Gap Theorem) Under the same assumption as Theorem 2, let $\Omega_{0}$ be a compactly contained subset of $\Omega$. Then, there exists $\epsilon_{0}>0$ such that $\operatorname{Ord}^{v}\left(x_{0}\right) \geq 1+\epsilon_{0}$ for all $x_{0} \in \mathcal{S}_{j}(u) \cap \Omega_{0}$.

By applying the above theorem for the case of higher order points (i.e. $j=$ 0 ), we obtain the following generalization of the $\epsilon$-gap theorem of GromovSchoen for DM-omplexes (cf. Theorem 6.3 of [GS]). 
Corollary 4 Let $\Omega$ be a Riemannian domain, $Y$ an NPC DM-complex, $u$ : $\Omega \rightarrow Y$ a harmonic map and $\Omega_{0}$ a compactly contained subset of $\Omega$. There exists $\epsilon_{0}>0$ such that if $x \in \Omega_{0}$, then either $\operatorname{Ord}^{u}(x)=1$ or $\operatorname{Ord}^{u}(x) \geq 1+\epsilon_{0}$.

In the follow-up article [DMV], we will show how to employ our main theorem in order to prove superrigidity for representations of lattices into new classes of groups not covered by [GS], for example isometry groups of hyperbolic buildings.

Acknowledgement This work was partially completed at the Max Planck Institute of Mathematics in Bonn. The authors would like to thank the Institute for their hospitality and generous support.

\section{Harmonic maps into NPC spaces and DM- complexes}

Recall that a metric space $(Y, d)$ is called an NPC space if:

(i) The space $(Y, d)$ is a length space. That is, for any two points $P$ and $Q$ in $Y$, there exists a rectifiable curve $c$ so that the length of $c$ is equal to $d(P, Q)$. We call such distance realizing curve a geodesic.

(ii) For any three points $P, R, Q \in Y$, let $c:[0, l] \rightarrow Y$ be the arclength parameterized geodesic from $Q$ to $R$ and let $Q_{t}=c(t l)$ for $t \in[0,1]$. Then

$$
d^{2}\left(P, Q_{t}\right) \leq(1-t) d^{2}(P, Q)+t d^{2}(P, R)-t(1-t) d^{2}(Q, R) .
$$

In particular, if $Y$ is an NPC space then between any two points $Q$ and $R$ the geodesic $c:[0,1] \rightarrow Y$ between them is unique. We use the notation

$$
Q_{t}=:(1-t) Q+t R
$$

We now define the notion of energy of a map to an NPC space $Y$. Let $\Omega$ be a smooth bounded $n$-dimensional Riemannian domain. A map $f: \Omega \rightarrow Y$ is said to be an $L^{2}$-map (or that $f \in L^{2}(\Omega, Y)$ ) if for some (and hence all) $P \in Y$, we have

$$
\int_{\Omega} d^{2}(f(x), P) d \mu<\infty .
$$


For $f \in L^{2}(\Omega, Y)$, define $e_{\epsilon}: \Omega \rightarrow \mathbf{R}$ by

$$
e_{\epsilon}(x)=\left\{\begin{array}{lc}
\int_{y \in \partial B_{\epsilon}(x)} \frac{d^{2}(f(x), f(y))}{\epsilon^{2}} d \Sigma & \text { for } x \in \Omega-N_{\epsilon}(\partial \Omega) \\
0 & \text { for } x \in N_{\epsilon}(\partial \Omega)
\end{array}\right.
$$

where $N_{\epsilon}(\partial \Omega)=\{x \in \Omega: d(x, \partial \Omega)<\epsilon\}$. Define a family of functionals

$$
E_{\epsilon}^{f}: C_{c}(\Omega) \rightarrow \mathbf{R}
$$

by setting

$$
E_{\epsilon}^{f}(\varphi)=\int_{\Omega} \varphi e_{\epsilon} d \mu
$$

We say $f$ has finite energy (or that $f \in W^{1,2}(\Omega, Y)$ ) if

$$
E^{f}:=\sup _{\varphi \in C_{c}(\Omega), 0 \leq \varphi \leq 1} \limsup _{\epsilon \rightarrow 0} E_{\epsilon}^{f}(\varphi)<\infty .
$$

It can be shown that if $f$ has finite energy, then the measures $e_{\epsilon}(x) d x$ converge as measures to a measure absolutely continuous with respect to the Lebesgue measure. Therefore there exists a function $e(x)$, which we call the energy density, such that $e_{\epsilon}(x) d \mu \rightarrow e(x) d \mu$. In analogy to the case of real valued functions, we write $|\nabla f|^{2}(x)$ in place of $e(x)$. In particular,

$$
E^{f}=\int_{\Omega}|\nabla f|^{2} d \mu
$$

If $f \in W^{1,2}(\Omega, Y)$, then there exists a well-defined notion of a trace of $f$, denoted $\operatorname{Tr}(f)$, which is an element of $L^{2}(\partial \Omega, Y)$. Two maps $f, g \in$ $W^{1,2}(\Omega, Y)$ have the same trace (i.e. $\left.\operatorname{Tr}(f)=\operatorname{Tr}(g)\right)$ if and only if $d(f, g) \in$ $W_{0}^{1,2}(\Omega)$. For details we refer to [KS1]. In the sequel, given $x \in \Omega$ and $f$ as above we will use the following notation

$$
E_{x}^{f}(\sigma):=\int_{B_{\sigma}(x)}|\nabla f|^{2} d \mu \text { and } I_{x}^{f}(\sigma):=\int_{\partial B_{\sigma}(x)} d^{2}(u, u(x)) d \Sigma(x) .
$$

Definition $5 A$ map $u: \Omega \rightarrow Y$ to an NPC space $Y$ is said to be harmonic if it is energy minimizing among all $W^{1,2}$-maps with the same boundary condition. 
The following regularity theorem is due to Gromov and Schoen [GS] and Korevaar and Schoen [KS1].

Theorem 6 A harmonic map $u: \Omega \rightarrow Y$ to an NPC space $Y$ is locally Lipschitz continuous with the local Lipschitz constant dependent only on the energy of $u$, the dimension of $\Omega$, the regularity of the metric $g$ of $\Omega$ and the distance to the boundary of $\Omega$.

Let $u: \Omega \rightarrow Y$ be a harmonic map. By Section 1.2 of [GS], there exists a constant $c>0$ depending only on the $C^{2}$ norm of the metric on $\Omega$ (in particular $c=0$ when $\Omega$ is Euclidean) such that

$$
\sigma \mapsto \operatorname{Ord}^{u}(x, \sigma):=e^{c \sigma^{2}} \frac{\sigma E_{x}^{u}(\sigma)}{I_{x}^{u}(\sigma)}
$$

is non-decreasing for any $x \in \Omega$. As a non-increasing limit of continuous functions,

$$
\operatorname{Ord}^{u}(x):=\lim _{\sigma \rightarrow 0} \operatorname{Ord}^{u}(x, \sigma)
$$

is an upper semicontinuous function. By following the proof of Theorem 2.3 in [GS], we see that $\operatorname{Ord}^{u}(x) \geq 1$. The value $\operatorname{Ord}^{u}(x)$ is called the order of $u$ at $x$.

Fix $x_{0} \in \Omega$ and choose a normal coordinate system centered at $x_{0}$. Without a loss of generality, we may write $x_{0}=0$ and assume $B_{1}(0)$ is contained in the normal neighborhood. Set $\alpha:=\operatorname{Ord}^{u}(0)$.

By Section 1.3 of [GS], there exists a constant $c>0$ such that

$$
\sigma \mapsto e^{c \sigma^{2}} \frac{I_{0}^{u}(\sigma)}{\sigma^{n-1+2 \alpha}}
$$

is monotone non-decreasing. Thus, if we set

$$
\mu_{\sigma}=\sqrt{\frac{I_{0}^{u}(\sigma)}{\sigma^{n-1}}}
$$

we see that

$$
\lim _{\sigma \rightarrow 0} \mu_{\sigma}=0 .
$$

For $\mu>0$, let $\mu^{-1} Y$ be the metric space $\left(Y, \mu^{-1} d\right)$. Set $g_{\sigma}(x)=g(\sigma x)$ and define

$$
u_{\sigma}: B_{\sigma^{-1}}(0) \rightarrow \mu_{\sigma}^{-1} Y
$$


by setting

$$
u_{\sigma}(x)=u(\sigma x) .
$$

By following Section 3 of [GS], we see that $u_{\sigma}$ is a harmonic map with $E_{0}^{u_{\sigma}}(1) \leq 2 \alpha$ and $I_{0}^{u_{\sigma}}(1)=1$. Let $g(0)$ be the Euclidean metric defined by the value of $g$ at 0 . By Theorem 2.4.6 of [KS1], $u_{\sigma}$ has a uniform modulus of continuity on compact sets independent of $\sigma$ (with respect to the metric $g(0)$ on the domain which is uniformly equivalent to $g_{\sigma}$ for $\sigma$ small). By [KS2], Proposition 3.7 and a diagonalization argument there exists $\sigma_{i} \rightarrow 0$ and a map $u_{*}: \mathbf{R}^{n} \rightarrow Y_{*}$ into an NPC space such that $u_{\sigma_{i}}$ converges to $u_{*}$ uniformly in the pull-back sense on every compact set. By (a slight modificaiton of) the $L^{2}$ trace theorem of [KS1], Theorem 1.12.2 and the fact that $I_{0}^{u_{\sigma}}(1)=1$, we have that $u_{*}$ is non-constant. Furthermore, by [KS2] Proposition 3.11 the energy of $u_{\sigma_{i}}$ converges to $u_{*}$ on compact subsets of $B_{1}(0)$ and $u_{*}$ is an energy minimizer again on compact subsets. We claim that $u_{*}$ is an energy minimizer on $B_{1}(0)$. Indeed, if $w:\left(B_{1}(0), g(0)\right) \rightarrow Y_{*}$ is an energy minimizing map with $\left.w\right|_{\partial B_{1}(0)}=\left.u_{*}\right|_{\partial B_{1}(0)}$, then Lemma 2.4.3 [KS1] implies that $d^{2}\left(u_{*}, w\right)$ is weakly subharmonic and hence $u_{*}=w$ on $B_{1}(0)$. Finally $u_{*}$ is instrinsically homogeneous degree $\alpha$, i.e.

$$
d\left(u_{*}(t x), u_{*}(0)\right)=t^{\alpha} d\left(u_{*}(x), u(0)\right) \text { for } 0 \leq t \leq 1, x \in \mathbf{R}^{n}
$$

by the same argument as in [GS] Proposition 3.3. Variations of the above argument will be used throughout the paper. Notice that in the above we do not need $Y$ to be locally compact. In the case when $Y$ is a locally compact simplicial complex, which is the main interest of this paper, the construction follows immediately from [GS] Proposition 3.3.

We now specialize our space $Y$ to be in a special class of cell complexes.

Definition 7 Let $\mathbf{E}^{d}$ be an affine space. A convex piecewise linear polyhedron $S$ with interior in some $\mathbf{E}^{i} \subset \mathbf{E}^{d}$ is called a cell. We will use the notation $S^{i}$ to indicate the dimension of $S$. A convex cell complex or simply a complex $Y$ in $\mathbf{E}^{d}$ is a finite collection $\mathcal{F}=\{S\}$ of cells satisfying the following properties: (i) the boundary $\partial S$ of $S^{i} \in \mathcal{F}$ is a union of $T^{j} \in \mathcal{F}$ with $j<i$ (called the faces of $S$ ) and (ii) if $T^{j}, S^{i} \in \mathcal{F}$ with $j<i$ and $S^{i} \cap T^{j} \neq \emptyset$, then $T^{j} \subset S^{i}$. For example a simplicial complex is a cell complex whose cells are all simplices. We will denote by $Y^{(i)}$ the i-dimensional skeleton of $Y$, i.e. 
the union of all cells $S^{j}$ where $j \leq i$. $Y$ is called $k$-dimensional or simply a $k$-complex if $Y^{(k+1)}=\emptyset$ but $Y^{(k)} \neq \emptyset$.

Definition 8 A complex $Y$ along with a metric $G=\left\{G^{S}\right\}$ is called a Riemannian complex if each cell $S$ of $Y$ is equipped with a smooth Riemannian metric $G^{S}$ such that for each cell $S$, the component functions of $G^{S}$ extend smoothly all the way to the boundary of $S$. Furthermore, if $S^{\prime}$ is a face of $S$ then the restriction $G^{S}$ to $S^{\prime}$ is equal to $G^{S^{\prime}}$ and $S^{\prime}$ is totally geodesic in $S$.

Throughout this paper, all cell complexes will have the additional property that all cells are bounded unless otherwise specified. If this is not the case, then we will write unbounded cell complex. Additionally, all cell complexes $Y$ will be locally compact, Riemannian and NPC with respect to the distance function $d$ induced from $G^{S}$.

Definition 9 We say a $k$-dimensional Riemannian complex $(Y, G)$ is a DMcomplex if given any two cells $S_{1}$ and $S_{2}$ of $Y$ such that $S_{1} \cap S_{2} \neq \emptyset$, there exists a $k$-dimensional $C^{\infty}$-differentiable complete Riemannian manifold $M$ and an isometric and totally geodesic embedding $J: M \rightarrow Y$ such that $S_{1} \cup S_{2} \subset J(M)$. By an abuse of notation, we will often denote $J(M)$ by $M$ and call it a DM (short for Differentiable Manifold).

Remark. If $Y$ is a Euclidean complex and we require that all the DM's to be isometric to a $k$-dimensional Euclidean space, then $Y$ is F-connected in the sense of [GS], Section 6.1.

Recall that for an arbitrary NPC space $Y$ and a point $P \in Y$, the Alexandrov tangent cone $T_{P} Y$ of $Y$ at $P$ is the cone over the space of directions $\Pi$. Here $\Pi$ is the completion of the space of equivalence classes of geodesics emanating from $P$ along with the distance function defined by the angle at $P$ where $\gamma_{1} \sim \gamma_{2}$ if the angle between them is equal to zero. For a DM-complex $Y$, let $C$ denote the tangent cone of $Y$ at the point $P$ as defined in [Fe] 3.1.21. Clearly, $C$ is an unbounded cell complex and $T_{P} Y$ is isometric to $(C, G(P))$ where $G(P)$ is the metric defined by the value of $G$ at $P$. Notice that if $P, Q \in \operatorname{int}(S)$, then $C$ for $P$ and $Q$ is the same set. Since $Y$ is piecewise smooth, we can consider the exponential map

$$
\exp _{P}^{Y}: B_{r}(0) \subset T_{P} Y \rightarrow B_{r}(P) \subset Y
$$


defined by piecing together the exponential maps defined on each cell containing $P$. This is equivalent to the exponential map defined from Alexandrov tangent cone point of view, i.e. given a unit speed geodesic $\gamma$ and $t \in[0, \infty)$, $\exp _{P}^{Y}(\gamma, t)=\gamma(t)$.

If $M$ is a DM passing through $P$ then define $F_{M}=T_{P} M \subset C$. An immediate consequence is the following.

Lemma 10 If $M$ is a DM in $(Y, G)$, then $F_{M}$ is a flat in $(C, G(P))=T_{P} Y$. In particular, if $Y$ is a DM-complex, then $T_{P} Y$ is F-connected in the sense of $[G S]$.

The next Lemma will be used in the sequel.

Lemma 11 Let $Y$ be a DM-complex, $u: \Omega \rightarrow Y$ a harmonic map and $\left.u_{*}:\left(B_{1}(0), g\left(x_{0}\right)\right)\right) \rightarrow Y_{*}$ a tangent map of $u$ at a point $x_{0} \in \Omega$. Then $Y_{*}$ is isometrically contained in $\left(C, G\left(u\left(x_{0}\right)\right)\right)=T_{u\left(x_{0}\right)} Y$.

Proof. Again we will choose normal coordinates around $x_{0}$ and identify $x_{0}=0$. Recall that by definition, the tangent map $u_{*}$ is the limit (in the pullback sense as in [KS2] Section 3) of the maps $u_{\sigma_{i}}: B_{1}(0) \rightarrow\left(C, \mu_{\sigma_{i}}^{-1} d\right)$ where $u_{\sigma_{i}}(x)=u\left(\sigma_{i} x\right)$. Consider the maps $\mu_{\sigma_{i}}{ }^{-1} u_{\sigma_{i}}: B_{1}(0) \rightarrow\left(C, G_{\sigma_{i}}\right)$ where $G_{\sigma_{i}}(y)=G\left(\mu_{\sigma_{i}} y\right)$. The two points of view are equivalent in the sense that the induced pullback pseudodistances on $B_{1}(0)$ are the same. Therefore we will work instead with the second point of view. The smoothness of the metric $G$ implies that $G_{\sigma_{i}}$ converges uniformly to the metric $G(u(0))$. Again, since $\mu_{\sigma_{i}}{ }^{-1} u_{\sigma_{i}}$ have uniformly bounded energy $E_{0}^{\mu_{\sigma_{i}}{ }^{-1} u_{\sigma_{i}}}(1)$ and uniformly bounded $I_{0}^{\mu_{\sigma_{i}}{ }^{-1} u_{\sigma_{i}}}(1)$, we obtain by Theorem 2.4 of [GS] and the ArzelaAscoli theorem that $\mu_{\sigma_{i}}{ }^{-1} u_{\sigma_{i}}$ converge uniformly on compact sets to a limit map $u_{0}:\left(B_{1}(0), g(0)\right) \rightarrow(C, G(u(0)))$, which by the equivalence of the two points of view it must be equal to the tangent map $u_{*}$. Q.E.D.

\section{Regular and Singular points}

As in the previous section, let $\Omega$ be an $n$-dimensional Riemannian domain and $(Y, G)$ a $k$-dimensional NPC DM-complex. 
Definition 12 Let $u: \Omega \rightarrow Y$ a harmonic map. A point $x_{0} \in \Omega$ is called a regular point if ord $^{u}\left(x_{0}\right)=1$ and there exists $\sigma_{0}>0$ such that

$$
u\left(B_{\sigma_{0}}\left(x_{0}\right)\right) \subset \exp _{u\left(x_{0}\right)}^{Y}\left(X_{0}\right),
$$

where $X_{0} \subset T_{u\left(x_{0}\right)} Y$ is isometric to $\mathbf{R}^{k}$. In particular, $x_{0}$ has a neighborhood mapping into a DM. A point $x_{0} \in \Omega$ is called a singular point if it is not a regular point. Denote the set of regular points by $\mathcal{R}(u)$ and the set of singular points by $\mathcal{S}(u)$.

Definition 13 Let $u: \Omega \rightarrow Y$ a harmonic map. Let

$$
\mathcal{S}_{0}(u)=\left\{x_{0} \in \Omega: \operatorname{Ord}^{u}\left(x_{0}\right)>1\right\} .
$$

Let $k_{0}:=\min \{n, k\}$ and $\mathcal{S}_{j}(u)=\emptyset$ for $j \notin\left\{1, \ldots, k_{0}\right\}$. For $j=1, \ldots, k_{0}$, we define $\mathcal{S}_{j}(u)$ inductively as follows. Having defined $\mathcal{S}_{m}(u)$ for $m=j+$ $1, \ldots, k_{0}+1$, define $\mathcal{S}_{j}(u)$ to be the set of points

$$
x_{0} \in \mathcal{S}(u) \backslash\left(\bigcup_{m=j+1}^{k_{0}} \mathcal{S}_{m}(u) \cup \mathcal{S}_{0}(u)\right)
$$

with the property that there exists $\sigma_{0}>0$ such that

$$
u\left(B_{\sigma_{0}}\left(x_{0}\right)\right) \subset \exp _{u\left(x_{0}\right)}^{Y}\left(X_{0}\right)
$$

where $X_{0} \subset T_{u\left(x_{0}\right)} Y$ is isometric to $\mathbf{R}^{j} \times Y_{2}^{k-j}$ and $Y_{2}$ is $(k-j)$-dimensional F-connected complex. Set

$$
\mathcal{S}_{m}^{-}(u)=\bigcup_{j=0}^{m} \mathcal{S}_{j}(u) \quad \text { and } \quad \mathcal{S}_{m}^{+}(u)=\bigcup_{j=m}^{k} \mathcal{S}_{j}(u)
$$

Lemma 14 The sets $\mathcal{S}_{0}(u), \mathcal{S}_{1}(u), \ldots, \mathcal{S}_{k_{0}-1}(u), \mathcal{S}_{k_{0}}(u)$ form a partition of $\mathcal{S}(u)$.

Proof. By definition, $\mathcal{S}_{0}(u), \ldots, \mathcal{S}_{k_{0}}(u)$ are mutually disjoint sets. Let $x_{0} \in \mathcal{S}(u)$. If $\operatorname{Ord}^{u}\left(x_{0}\right)>1$, then $x_{0} \in \mathcal{S}_{0}(u)$. If $\operatorname{Ord}^{u}\left(x_{0}\right)=1$, then the tangent map $u_{*}: \mathbf{R}^{n} \rightarrow T_{u\left(x_{0}\right)} Y$ at $x_{0}$ is a homogeneous degree 1 map and maps onto a flat $F_{0} \subset T_{u\left(x_{0}\right)} Y$ by Proposition 3.1 of [GS]. Let 
$X_{0}$ be the union of all $k$-flats containing $F_{0}$. By Lemma 6.2 of [GS], $X_{0}$ is isometric to $\mathbf{R}^{j} \times Y_{2}^{k-j}$ where $j \in\left\{1, \ldots, k_{0}\right\}$ is the dimension of $F_{0}$. Furthermore, by the same lemma, $u_{*}$ is effectively contained in $X_{0}$. Since $\sup _{B_{r}\left(x_{0}\right)} d\left(u, \exp _{u\left(x_{0}\right)}^{Y} \circ u_{*} \circ\left(\exp _{x_{0}}^{\Omega}\right)^{-1}\right) \rightarrow 0$ as $r \rightarrow 0$, this implies by Theorem 5.1 of $[\mathrm{GS}]$ that $x_{0} \in \mathcal{S}_{j}^{+}(u)$ and hence $x_{0} \in \mathcal{S}_{m}(u)$ for some $m \in\left\{j, \ldots, k_{0}\right\}$. Q.E.D.

Lemma 15 The sets $\mathcal{R}(u), \mathcal{R}(u) \cup \mathcal{S}_{m}^{+}(u)$ are open and the sets $\mathcal{S}_{m}^{-}(u)$ are closed.

Proof. Clearly $\mathcal{R}(u)$ and $\mathcal{R}(u) \cup \mathcal{S}_{0}^{+}(u)=\Omega$ are open. Now assume $m>0$ and $x_{0} \in \mathcal{S}_{m}^{+}(u)$. Thus, $x_{0} \in \mathcal{S}_{j}(u)$ for an integer $j \geq m$, hence $\operatorname{Ord}^{u}\left(x_{0}\right)=1$ and there exists $\sigma_{0}>0$ such that $u\left(B_{\sigma_{0}}\left(x_{0}\right)\right) \subset \exp _{u\left(x_{0}\right)}^{Y}\left(X_{0}\right)$ where $X_{0}$ is isometric to $\mathbf{R}^{j} \times Y_{2}^{k-j}$. Thus, $x \in B_{\sigma}\left(x_{0}\right)$ implies $x \in \mathcal{S}^{l}(u) \cup$ $\mathcal{R}(u)$ for some $l \in\left\{j, \ldots, k_{0}\right\}$, i.e $x \in \mathcal{S}_{m}^{+}(u) \cup \mathcal{R}(u)$. This shows $\mathcal{S}_{m}^{+}(u) \cup \mathcal{R}(u)$ is open which in turn this implies $\mathcal{S}_{m}^{-}(u)=\Omega \backslash\left(\mathcal{S}_{m+1}^{+}(u) \cup \mathcal{R}(u)\right)$ is closed. Q.E.D.

\section{Near a singular point $x \in \mathcal{S}_{j}(u)$ where $j>0$}

Let $u: \Omega \rightarrow(Y, G)$ be a harmonic map and $x_{\star} \in \mathcal{S}_{j}(u), j>0$. As a degree 1 homogeneous map, the tangent map $u_{*}: \mathbf{R}^{n} \rightarrow\left(C, G\left(u\left(x_{\star}\right)\right)\right)$ at $x_{\star}$ is effectively is contained in a subcomplex isometric a product $\mathbf{R}^{j} \times Y_{2}^{k-j}$ of Euclidean space $\mathbf{R}^{j}$ and an $(k-j)$-dimensional F-connected complex $Y_{2}$ with a single vertex $P_{0}$ by [GS] Lemma 6.2. For convenience, identify $\mathbf{R}^{j} \times Y_{2}^{k-j}$ with its isometric image in $\left(C, G\left(u\left(x_{\star}\right)\right)\right)$. Note then that $\mathbf{R}^{j} \times Y_{2}^{k-j}$ is the union of all $k$-flats containing the $j$-flat $\mathbf{R}^{j} \times\left\{P_{0}\right\}$ (cf. [GS] Lemma 6.2) which we write as

$$
\mathbf{R}^{j} \times Y_{2}^{k-j}=\bigcup_{i=1}^{L} F_{i} .
$$

Conversely, every $k$-flat of $\mathbf{R}^{j} \times Y_{2}^{k-j}$ is one of $\left\{F_{i}\right\}_{i=1}^{L}$. To see this, note that if $F$ is a $k$-flat in $\mathbf{R}^{j} \times Y_{2}^{k-j}$ then $\pi_{1}(F)$ and $\pi_{2}(F)$ are flats in $\mathbf{R}^{j}$ and $Y_{2}^{k-j}$ respectively where $\pi_{1}$ and $\pi_{2}$ are the projections onto the two factors $\mathbf{R}^{j}$ and $Y_{2}^{k-j}$. Since $\operatorname{dim}\left(\pi_{1}(F)\right)+\operatorname{dim}\left(\pi_{2}(F)\right)=\operatorname{dim}(F)=k$, we necessarily 
have $\operatorname{dim}\left(\pi_{1}(F)\right)=j$ and $\operatorname{dim}\left(\pi_{2}(F)\right)=k-j$. Thus $\pi_{1}(F)=\mathbf{R}^{j}$ and, since $\mathbf{R}^{j} \times Y_{2}^{k-j}$ is a cone (as an image of the homogeneous map $u_{*}$ ), $\pi_{2}(F)$ must contain the point $P_{0}$. This implies that $F$ contains the $j$-flat $\mathbf{R}^{j} \times\left\{P_{0}\right\}$.

The metric $G\left(u\left(x_{\star}\right)\right)$ restricted to $\mathbf{R}^{j} \times Y_{2}^{k-j}$ is a product metric which we write as $H \times h$. In particular, $\left(\mathbf{R}^{j}, H\right)$ is a Euclidean space and $\left(Y_{2}^{k-j}, h\right)$ is a $(k-j)$-dimensional F-connected NPC complex as in [GS]. Consider the metric defined in a neighborhood $U$ of $u\left(x_{\star}\right)$ in $\mathbf{R}^{j} \times Y_{2}^{k-j}$ by pulling back the metric $G$ via the map $\exp _{u\left(x_{\star}\right)}^{Y}$ and denote it $G$ by an abuse of notation. Then $\left(U \cap F_{i},\left.G\right|_{U \cap F_{i}}\right)$ is a $k$-dimensional differentiable manifold for any $F_{i}$ as in (5). Conversely, if $\left(V,\left.G\right|_{V}\right)$ is a $k$-dimensional differentiable manifold where $u\left(x_{\star}\right) \in V \subset U$, then $\left(V, G\left(u\left(x_{\star}\right)\right)\right)$ is isometric to a $k$-dimensional Euclidean domain and hence $V \subset F_{i}$. We extend $G$ to all of $\mathbf{R}^{j} \times Y_{2}^{k-j}$ so that the elements of $\left\{\left(F_{i},\left.G\right|_{F_{i}}\right)\right\}$ are the DM's of $\left(\mathbf{R}^{j} \times Y_{2}^{k-j}, G\right)$. Then $\left(\mathbf{R}^{j} \times Y_{2}^{k-j}, G\right)$ is a DM-connected complex and every DM of $\left(\mathbf{R}^{j} \times Y_{2}^{k-j}, G\right)$ has the form $M=\left(F_{i},\left.G\right|_{F_{i}}\right)$ for $F_{i}$.

Since we are only interested in the local properties of $u$, in this section as well as in the sections that follow, we will consider a harmonic map

$$
u:\left(B_{\sigma_{\star}}\left(x_{\star}\right), g\right) \rightarrow\left(\mathbf{R}^{j} \times Y_{2}^{k-j}, G\right)
$$

with

$$
V:=\pi_{1} \circ u: B_{\sigma_{\star}}\left(x_{\star}\right) \rightarrow \mathbf{R}^{j} \text { and } v:=\pi_{2} \circ u: B_{\sigma_{\star}}\left(x_{\star}\right) \rightarrow Y_{2}^{k-j} .
$$

We note that $v(x)=P_{0}$ for any $x \in \mathcal{S}_{j}(u)$.

Lemma 16 Let $u_{*}:\left(B_{1}(0), g(0)\right) \rightarrow\left(\mathbf{R}^{j} \times Y_{2}^{k-j}, G(x)\right)$ be a tangent map of $u$ at $x \in \mathcal{S}_{j}(u)$. Then $v_{*}:=\pi_{2} \circ u_{*}=0$.

Proof. Assume on the contrary that $v_{*} \neq 0$. Since $u_{*}$ is a homogeneous degree 1 map, so is $v_{*}$. Thus $v_{*}$ maps into a flat $F_{0}$ of $Y_{2}^{k-j}$ by Proposition 3.1 of [GS]. Let $X_{0}$ be the union of all $(k-j-l)$-flats containing $F_{0}$ where $l$ is the dimension of $F_{0}$. By Lemma 6.2 of [GS], $X_{0}$ is isometric to $\mathbf{R}^{j+l} \times Z_{2}^{k-j-l}$ and $u_{*}$ is effectively contained in $\mathbf{R}^{j+l} \times Z_{2}^{k-j-l}$. Since $\sup _{B_{r}(x)} d\left(u, \exp _{u(x)}^{Y} \circ u_{*} \circ\left(\exp _{x}^{\Omega}\right)^{-1}\right) \rightarrow 0$ as $r \rightarrow 0$, this implies that $x \in \mathcal{S}_{j+l}^{+}$ by Theorem 5.1 of [GS] which contradicts that $x \in \mathcal{S}_{j}(u)$. Q.E.D. 
Lemma 17 Let $\eta \in C_{c}^{\infty}\left(B_{\sigma_{\star}}\left(x_{\star}\right)\right)$. Considering $v$ as a map into the $N P C$ space $\left(Y_{2}^{k-j}, h\right)$, set

$$
v_{\eta}(x)=(1-\eta(x)) v(x)+\eta(x) P_{0} \quad \text { and } \quad u_{\eta}=\left(V, v_{\eta}\right) .
$$

Then

$$
|\nabla u|^{2}(x)=\left|\nabla u_{\eta}\right|^{2}(x), \text { a.e. } x \in \mathcal{S}_{j}(u) .
$$

Furthermore,

$$
|\nabla v|^{2}(x)=\left|\nabla v_{\eta}\right|^{2}(x)=0, \text { a.e. } x \in \mathcal{S}_{j}(u)
$$

in other words, the energy density functions of $v$ and $v_{\eta}$ as maps into $\left(Y_{2}^{k-j}, h\right)$ agree on $\mathcal{S}_{j}(u)$.

Proof. Let $\iota:\left(\mathbf{R}^{j} \times Y_{2}^{k-j}, G\right) \hookrightarrow \mathbf{R}^{N}$ be an isometric embedding. Let $x_{0} \in \mathcal{S}_{j}(u)$ be a Lebesgue point of

$$
\left|\nabla\left(\iota \circ\left(V, P_{0}\right)\right)\right|^{2}=\sum_{\alpha, \beta} g^{\alpha \beta}<\frac{\partial\left(\iota \circ\left(V, P_{0}\right)\right)}{\partial x^{\alpha}}, \frac{\partial\left(\iota \circ\left(V, P_{0}\right)\right)}{\partial x^{\beta}}>
$$

and

$$
|\nabla(\iota \circ u)|^{2}=\sum_{\alpha, \beta} g^{\alpha \beta}<\frac{\partial(\iota \circ u)}{\partial x^{\alpha}}, \frac{\partial(\iota \circ u)}{\partial x^{\beta}}>.
$$

Furthermore, since $\iota \circ u: B_{\sigma}\left(x_{0}\right) \rightarrow \mathbf{R}^{N}$ and $\iota \circ\left(V, P_{0}\right): B_{\sigma}\left(x_{0}\right) \rightarrow \mathbf{R}^{N}$ are Lipschitz by Theorem 6 and hence differentiable a.e., we can assume

$$
\frac{\partial(\iota \circ u)}{\partial x^{\alpha}}\left(x_{0}\right) \text { and } \frac{\partial\left(\iota \circ\left(V, P_{0}\right)\right)}{\partial x^{\alpha}}\left(x_{0}\right) \text { exist } \forall \alpha=1, \ldots, n \text {. }
$$

Using normal coordinates, identify $x_{0}=0$ and let $g_{\sigma}(x)=g(\sigma x)$. Furthermore, we can assume that $\iota \circ u(0)=0 \in \mathbf{R}^{N}$. By pulling back the metric $G$ to $T_{0}\left(\iota\left(\mathbf{R}^{j} \times Y_{2}^{k-2}\right)\right) \subset \mathbf{R}^{N}$ via the map $\exp _{0} \circ \iota$, we can define a metric near the vertex which we call $G$ again by an abuse of notation. Define $G_{\sigma}(y)=\mu_{\sigma}^{-1} G(y)$ and note that $G_{\sigma}$ converges uniformly on every compact set to the metric $G(0)$. Note that $G(0)$ is the restriction of the standard inner product $<\cdot, \cdot>$ of $\mathbf{R}^{N}$ to $T_{0}\left(\iota\left(\mathbf{R}^{j} \times Y_{2}^{k-j}\right)\right)$. Since $T_{0}\left(\iota\left(\mathbf{R}^{j} \times\left\{P_{0}\right\}\right)\right)$ is a $j$ dimensional linear space in $\mathbf{R}^{N}$, by rotating if necessary, we may assume it to be equal to the first factor $\mathbf{R}^{j}$ of $\mathbf{R}^{j} \times \mathbf{R}^{N-j}=\mathbf{R}^{N}$. We use $\hat{V}=\left(\hat{V}^{1}, \ldots, \hat{V}^{j}\right)$ 
and $\hat{v}=\left(\hat{v}^{1}, \ldots, \hat{v}^{N}\right)$ as coordinates of $\mathbf{R}^{j} \times \mathbf{R}^{N-j}$. Let $\hat{\pi}_{1}$ and $\hat{\pi}_{2}$ denote the orthogonal projections with respect to the metric $G(0)$ onto the subspaces $\mathbf{R}^{j}$ and $\mathbf{R}^{N-j}$ respectively of $\mathbf{R}^{N}$. Using this, we can write

$$
\hat{u}:=\exp _{0}^{-1} \circ \iota \circ u=(\hat{V}, \hat{v}): B_{1}(0) \rightarrow \mathbf{R}^{j} \times \mathbf{R}^{N-j}=\mathbf{R}^{N}
$$

where

$$
\hat{V}=\hat{\pi}_{1} \circ \hat{u} \text { and } \hat{v}=\hat{\pi}_{2} \circ \hat{u} .
$$

Similarly for the blow up maps $\hat{u}_{\sigma}$ of $\hat{u}$ we can write

$$
\hat{u}_{\sigma}=\left(\hat{V}_{\sigma}, \hat{v}_{\sigma}\right): B_{1}(0) \rightarrow \mathbf{R}^{j} \times \mathbf{R}^{N-j}=\mathbf{R}^{N} .
$$

Note that by construction,

$$
d \iota_{\left(0, P_{0}\right)} \circ\left(\pi_{1}, 0\right)=\left(\hat{\pi}_{1} \circ d \iota_{\left(0, P_{0}\right)}, 0\right) .
$$

By the smoothness of the metric $G$, the fact that $\exp _{0}$ is $C^{\infty}$ close to the identity map near 0 and (7) we have that

$$
\begin{aligned}
g^{\alpha \beta} & <\frac{\partial\left(\iota \circ\left(V, P_{0}\right)\right)}{\partial x^{\alpha}}, \frac{\partial\left(\iota \circ\left(V, P_{0}\right)\right)}{\partial x^{\beta}}>(x) \\
& =g^{\alpha \beta}<\frac{\partial\left(\iota \circ\left(\pi_{1}, P_{0}\right) \circ u\right)}{\partial x^{\alpha}}, \frac{\partial\left(\iota \circ\left(\pi_{1}, P_{0}\right) \circ u\right)}{\partial x^{\beta}}>(x) \\
& =(1+O(|x|)) g^{\alpha \beta}<\frac{\partial\left(\hat{\pi}_{1} \circ \iota \circ u\right)}{\partial x^{\alpha}}, \frac{\partial\left(\hat{\pi}_{1} \circ \iota \circ u\right)}{\partial x^{\beta}}>(x) \\
& =(1+O(|x|)) g^{\alpha \beta}<\frac{\partial\left(\hat{\pi}_{1} \circ \exp _{0}^{-1} \circ \iota \circ u\right)}{\partial x^{\alpha}}, \frac{\partial\left(\hat{\pi}_{1} \circ \exp _{0}^{-1} \circ \iota \circ u\right)}{\partial x^{\beta}}>(x) \\
& =(1+O(|x|)) g^{\alpha \beta}<\frac{\partial \hat{V}}{\partial x^{\alpha}}, \frac{\partial \hat{V}}{\partial x^{\beta}}>(x)
\end{aligned}
$$

and

$$
\begin{aligned}
g^{\alpha \beta} & <\frac{\partial(\iota \circ u)}{\partial x^{\alpha}}, \frac{\partial(\iota \circ u)}{\partial x^{\beta}}>(x) \\
& =(1+O(|x|)) g^{\alpha \beta}<\frac{\partial\left(\exp _{0}^{-1} \circ \iota \circ u\right)}{\partial x^{\alpha}}, \frac{\partial\left(\exp _{0}^{-1} \circ \iota \circ u\right)}{\partial x^{\beta}}>(x) \\
& =(1+O(|x|)) g^{\alpha \beta}<\frac{\partial \hat{u}}{\partial x^{\alpha}}, \frac{\partial \hat{u}}{\partial x^{\beta}}>(x) .
\end{aligned}
$$


Let $\hat{u}_{\sigma_{i}}$ be the sequence converging uniformly in the pull back sense to a tangent map

$$
u_{*}=\left(V_{*}, v_{*}\right): B_{1}(0) \rightarrow\left(T_{0}\left(\iota\left(\mathbf{R} \times Y_{2}^{k-j}\right)\right), G(u(0))\right) \hookrightarrow \mathbf{R}^{j} \times \mathbf{R}^{N-j}
$$

and ${ }^{G_{\sigma_{i}}} E^{\hat{u}_{\sigma_{i}}}(r) \rightarrow{ }^{G(0)} E^{u_{*}}(r)$ for $r \in(0,1)$ (cf. Theorem 3.11 [KS2]). In fact, because of the uniform convergence $G_{\sigma}$ to $G(0)$, we have ${ }^{G(0)} E^{\hat{u}_{\sigma_{i}}}(r) \rightarrow$ ${ }^{G(0)} E^{u_{*}}(r)$ for $r \in(0,1)$. Recalling that $G(0)$ is the restriction of the standard Euclidean metric $<\cdot, \cdot>$ on $\mathbf{R}^{N}$ to $T_{0}\left(\iota\left(\mathbf{R} \times Y_{2}^{k-j}\right)\right)$, we can write this as

$$
\int_{B_{r}(0)} \sum_{\alpha, \beta} g^{\alpha \beta}<\frac{\partial u_{*}}{\partial x^{\alpha}}, \frac{\partial u_{*}}{\partial x^{\beta}}>d \mu=\lim _{i \rightarrow \infty} \int_{B_{r}(0)} \sum_{\alpha, \beta} g^{\alpha \beta}<\frac{\partial \hat{u}_{\sigma_{i}}}{\partial x^{\alpha}}, \frac{\partial \hat{u}_{\sigma_{i}}}{\partial x^{\beta}}>d \mu .
$$

Since $\hat{u}_{\sigma_{i}}$ converges uniformly to $u_{*}$ in $B_{r}(0), \hat{V}_{\sigma_{i}}$ converges uniformly to $V_{*}$ in $B_{r}(0)$ and $\hat{v}_{\sigma_{i}}$ converges uniformly to $v_{*}$. We additionally have that $\hat{V}_{\sigma_{i}}$ and $\hat{v}_{\sigma_{i}}$ are uniformly Lipschitz in $B_{r}(1)$ for any $r \in(0,1)$. Thus, the lower semicontinuity of energy (cf. [KS1] Theorem 1.6.1) implies

$$
\begin{aligned}
& \int_{B_{r}(0)} \sum_{\alpha, \beta} g^{\alpha \beta}<\left(\frac{\partial V_{*}}{\partial x^{\alpha}}, 0\right),\left(\frac{\partial V_{*}}{\partial x^{\beta}}, 0\right)>d \mu \\
& \leq \lim _{i \rightarrow \infty} \int_{B_{r}(0)} \sum_{\alpha, \beta} g^{\alpha \beta}<\left(\frac{\partial \hat{V}_{\sigma_{i}}}{\partial x^{\alpha}}, 0\right),\left(\frac{\partial \hat{V}_{\sigma_{i}}}{\partial x^{\beta}}, 0\right)>d \mu
\end{aligned}
$$

and

$$
\begin{aligned}
& \int_{B_{r}(0)} \sum_{\alpha, \beta} g^{\alpha \beta}<\left(0, \frac{\partial v_{*}}{\partial x^{\alpha}}\right),\left(0, \frac{\partial v_{*}}{\partial x^{\beta}}\right)>d \mu \\
& \quad \leq \lim _{i \rightarrow \infty} \int_{B_{r}(0)} \sum_{\alpha, \beta} g^{\alpha \beta}<\left(0, \frac{\partial \hat{v}_{\sigma_{i}}}{\partial x^{\alpha}}\right),\left(0, \frac{\partial \hat{v}_{\sigma_{i}}}{\partial x^{\beta}}\right)>d \mu .
\end{aligned}
$$

On the other hand, we can write

$$
<\frac{\partial \hat{u}_{\sigma_{i}}}{\partial x^{\alpha}}, \frac{\partial \hat{u}_{\sigma_{i}}}{\partial x^{\beta}}>=<\left(\frac{\partial \hat{V}_{\sigma_{i}}}{\partial x^{\alpha}}, 0\right),\left(\frac{\partial \hat{V}_{\sigma_{i}}}{\partial x^{\beta}}, 0\right)>+<\left(0, \frac{\partial \hat{v}_{\sigma_{i}}}{\partial x^{\alpha}}\right),\left(0, \frac{\partial \hat{v}_{\sigma_{i}}}{\partial x^{\beta}}\right)>
$$

and

$$
<\frac{\partial u_{*}}{\partial x^{\alpha}}, \frac{\partial u_{*}}{\partial x^{\beta}}>=<\left(\frac{\partial V_{*}}{\partial x^{\alpha}}, 0\right),\left(\frac{\partial V_{*}}{\partial x^{\beta}}, 0\right)>+<\left(0, \frac{\partial v_{*}}{\partial x^{\alpha}}\right),\left(0, \frac{\partial v_{*}}{\partial x^{\beta}}\right)>.
$$


Thus, the above two equalities with (10), (11) and (12) implies that we have equalities in (11) and (12). The fact that $0 \in \mathcal{S}_{j}(u)$ implies $u_{*}(0)=\left(V_{*}(0), 0\right)$ by Lemma 16 and hence

$$
\lim _{i \rightarrow \infty} \int_{B_{r}(0)} \sum_{\alpha, \beta} g^{\alpha \beta}<\left(0, \frac{\partial \hat{v}_{\sigma_{i}}}{\partial x^{\alpha}}\right),\left(0, \frac{\partial \hat{v}_{\sigma_{i}}}{\partial x^{\beta}}\right)>d \mu=0
$$

and

$$
\begin{gathered}
\lim _{i \rightarrow \infty} \int_{B_{r}(0)} \sum_{\alpha, \beta} g^{\alpha \beta}<\left(\frac{\partial \hat{V}_{\sigma_{i}}}{\partial x^{\alpha}}, 0\right),\left(\frac{\partial \hat{V}_{\sigma_{i}}}{\partial x^{\beta}}, 0\right)>d \mu \\
=\lim _{i \rightarrow \infty} \int_{B_{r}(0)} \sum_{\alpha, \beta} g^{\alpha \beta}<\frac{\partial \hat{u}_{\sigma_{i}}}{\partial x^{\alpha}}, \frac{\partial \hat{u}_{\sigma_{i}}}{\partial x^{\beta}}>d \mu .
\end{gathered}
$$

Using the fact that 0 is a Lebesgue point of $\mid\left.\nabla\left(\left.\iota \circ\left(V, P_{0}\right)\right|^{2}\right.$ and $\left.\mid \nabla(\iota \circ u)\right)\right|^{2}$ and equations (8) and (9), we see that

$$
\begin{aligned}
\frac{\left|\nabla\left(\iota \circ\left(V, P_{0}\right)\right)\right|^{2}(0)}{|\nabla(\iota \circ u)|^{2}(0)} & =\frac{\lim _{i \rightarrow \infty} \int_{B_{r}(0)} \sum_{\alpha, \beta} g^{\alpha \beta}<\left(\frac{\partial \hat{V}_{\sigma_{i}}}{\partial x^{\alpha}}, 0\right),\left(\frac{\partial \hat{V}_{\sigma_{i}}}{\partial x^{\beta}}, 0\right)>d \mu}{\lim _{i \rightarrow \infty} \int_{B_{r}(0)} \sum_{\alpha, \beta} g^{\alpha \beta}<\frac{\partial \hat{u}_{\sigma_{i}}}{\partial x^{\alpha}}, \frac{\partial \hat{u}_{\sigma_{i}}}{\partial x^{\beta}}>d \mu} \\
& =1 .
\end{aligned}
$$

In the above equality, the fact that the denominator is non-zero follows from the fact that $0 \notin \mathcal{S}_{0}(u)$. Therefore, we have shown $\left|\nabla\left(\iota \circ\left(V, P_{0}\right)\right)\right|^{2}(0)=$ $|\nabla(\iota \circ u)|^{2}(0)$ for a.e. $x \in \mathcal{S}_{j}(u)$, which implies

$$
\frac{\partial(\iota \circ(V(0), v))}{\partial x^{\alpha}}(0)=0, \forall \alpha=1, \ldots, n .
$$

Taking into account that $v(0)=P_{0}$, we have

$$
d^{2}\left(v_{\eta}(0), v_{\eta}(y)\right)=\eta^{2}(y) d^{2}(v(0), v(y)) .
$$

Using $|\nabla v|^{2}$ and $\left|\nabla v_{\eta}\right|^{2}$ to denote the energy density functions of $v$ and $v_{\eta}$ with respect to $h$, we obtain from (14) the following:

$$
(13) \Rightarrow|\nabla v|^{2}(0)=0
$$




$$
\Rightarrow\left|\nabla v_{\eta}\right|^{2}(0)=0 \Rightarrow \frac{\partial\left(\iota \circ\left(V(0), v_{\eta}\right)\right)}{\partial x^{\alpha}}(0)=0, \forall \alpha=1, \ldots, n .
$$

Thus,

$$
|\nabla(\iota \circ u)|^{2}\left(x_{0}\right)=\left|\nabla\left(\iota \circ\left(V, P_{0}\right)\right)\right|^{2}\left(x_{0}\right)=\left|\nabla\left(\iota \circ u_{\eta}\right)\right|^{2}\left(x_{0}\right),
$$

which proves the Lemma. Q.E.D.

We will now derive estimates of the metric $G$ defined on $\mathbf{R}^{j} \times Y_{2}^{k-j}$ near $\left(0, P_{0}\right)$. Let $V=\left(V^{1}, \ldots, V^{j}\right)$ be the standard coordinates on $\mathbf{R}^{j}$ and $v=$ $\left(v^{j+1}, \ldots, v^{k}\right)$ the standard coordinates on $\mathbf{R}^{k-j}$. We combine $(V, v)$ to denote the standard coordinates on $\mathbf{R}^{k}$.

Let $M$ be any DM of $\left(\mathbf{R}^{j} \times Y_{2}^{k-j}, G\right)$. Note that $M=\left(F_{i},\left.G\right|_{F_{i}}\right)$ for $F_{i}$ as in (5). By assumption $\mathbf{R}^{j} \times\left\{P_{0}\right\}$ is totally geodesic in $M$ and thus there exist orthonormal coordinates

$$
\psi: U_{0} \subset \mathbf{R}^{j} \rightarrow \mathbf{R}^{j} \times\left\{P_{0}\right\} \subset M, \psi(0)=\left(0, P_{0}\right)
$$

at 0 . Let

$$
V \mapsto e_{j+1}(V, 0), \ldots, V \mapsto e_{k}(V, 0)
$$

be vector fields along $\psi$ such that at each $V,\left\{e_{l}(V, 0)\right\}$ forms an orthonormal basis of the normal space to $\psi\left(U_{0}\right)$ in $M$ at $\psi(V)$.

We next identify, by the tubular neighborhood theorem, a neigborhood $U$ of $P_{0}$ in $M$ with a disc bundle over $\psi\left(U_{0}\right)$, where the fiber over $\psi(V)$ is denoted by $\Pi_{V}$. Let $G_{V}$ denote the restriction of $G$ to $\Pi_{V}$. The existence of normal coordinates of $\left(\Pi_{V}, G_{V}\right)$ implies that we can define a diffeomorphism

$$
\phi_{V}: \mathcal{U}_{V} \subset \mathbf{R}^{k-j} \rightarrow \phi_{V}\left(U_{V}\right) \subset \Pi_{V}
$$

such that

$$
\phi_{V}(0)=\psi(V)
$$

and

$$
\nabla_{e_{l}} e_{m}(V, 0)=0
$$

where

$$
e_{l}(V, v)=\phi_{V *}\left(\frac{\partial}{\partial v^{l}}\right)(v) \text { for } l=j+1, \ldots, k
$$


can be chosen to agree with the previous definition at $V=0$. Now define a diffeomorphism

$$
\Phi: \mathcal{U} \subset \mathbf{R}^{k} \rightarrow \Phi(\mathcal{U}) \subset U \text { by } \Phi(V, v)=\phi_{V}(v)
$$

for $\mathcal{U}$ a sufficiently small neighborhood of $0 \in \mathbf{R}^{k}$. Thus, $\Phi(V, 0)=\psi(V)$ and

$$
e_{l}(V, v)=\Phi_{*}\left(\frac{\partial}{\partial v^{l}}\right)(V, v) \text { for } l=j+1, \ldots, k .
$$

Let

$$
E_{I}(V, v)=\Phi_{*}\left(\frac{\partial}{\partial V^{I}}\right)(V, v) \text { for } I=1, \ldots, j .
$$

Lemma 18 Let $M$ be a $D M$ in $\left(\mathbf{R}^{j} \times Y_{2}^{k-j}, G\right)$ through $\left(0, P_{0}\right)$ and $\Phi: \mathcal{U} \subset$ $\mathbf{R}^{j} \times \mathbf{R}^{k-j} \rightarrow M$ be the coordinates defined above with $\Phi(0,0)=\left(0, P_{0}\right)$. Let

$$
G=\left(\begin{array}{ll}
G_{11}(V, v) & G_{12}(V, v) \\
G_{21}(V, v) & G_{22}(V, v)
\end{array}\right)
$$

where

$$
\begin{aligned}
& G_{11}(V, v)=\left(G_{11}(V, v)_{I J}\right) \quad G_{12}(V, v)=\left(G_{12}(V, v)_{I l}\right) \\
& G_{21}(V, v)=\left(G_{21}(V, v)_{l I}\right) \quad G_{22}(V, v)=\left(G_{22}(V, v)_{l m}\right)
\end{aligned}
$$

is the matrix representation of $G$ in the coordinates $\Phi$ defined above with $I, J=1, \ldots, j$ and $l, m=j+1, \ldots, k$. Then for any $(V, 0) \in \mathcal{U}$,

$$
G_{11}(0,0)=I \text { and } G_{22}(V, 0)=I,
$$

where $I$ is the identity matrix. Furthermore,

$$
\begin{array}{ll}
\left|G_{11}(V, v)_{I J}-G_{11}(V, 0)_{I J}\right| \leq C|v|^{2}, & \left|\frac{\partial}{\partial v^{l}} G_{11}(V, v)_{I J}\right| \leq C|v| \\
\left|G_{22}(V, v)_{l m}-G_{22}(V, 0)_{l m}\right| \leq C|v|^{2}, & \left|\dot{G}_{22}(V, v)_{l m}\right| \leq C|v| \\
\left|G_{12}(V, v)_{I l}\right|<C|v|^{2}, & \left|\dot{G}_{12}(V, v)_{I l}\right| \leq C|v| .
\end{array}
$$

In the above $\dot{G}$ is used indicate any derivatives (i.e. $\frac{\partial}{\partial V^{I}}$ or $\frac{\partial}{\partial v^{l}}$ ) and $|v|$ is the radial component of the normal coordinates on plane $\Pi_{V}$ and hence invariantly defined. Furthermore,

$$
|v| \leq C d\left(v(x), P_{0}\right)=: C d_{P_{0}}(x)
$$

where $d$ is the distance function defined on $\left(Y_{2}^{k-j}, h\right)$ and the constant $C$ depends only on the $C^{2}$ norm of the metric $G$. 
Proof. The equality $G_{11}(0,0)=I$ follows from the fact that the restriction of $\Phi$ to $U_{0} \times\{0\} \cap \mathcal{U}$ is the orthonormal coordinates $\psi$ of (15). The equality $G_{22}(V, 0)=I$ for any $(V, 0) \in \mathcal{U}$ follows from the fact that the restriction of $\Phi$ to $\{V\} \times \mathcal{U}_{V} \cap \mathcal{U}$ is the orthonormal coordinates $\phi_{V}$ of (16). This proves (18).

To prove (19), it suffices to prove the following properties:

$$
\begin{aligned}
& \text { (i) }<E_{I}, e_{l}>(V, 0)=0 \\
& \text { (ii) } E_{J}<E_{I}, e_{l}>(V, 0)=0 \\
& \text { (iii) } e_{m}<E_{I}, e_{l}>(V, 0)=0 \\
& \text { (iv) } e_{m}<E_{I}, E_{J}>(V, 0)=0 \\
& \text { (v) } E_{I}<e_{l}, e_{m}>(V, 0)=0 \\
& \text { (vi) } e_{m}<e_{l}, e_{p}>(V, 0)=0 \text {. }
\end{aligned}
$$

Indeed, $(i)$ follows from the fact that $\Phi$ maps $\{(V, 0) \in \mathcal{U}\}$ to $\psi\left(U_{0}\right)$ and $e_{l}(V, 0)$ is chosen to be normal to $\psi\left(U_{0}\right)$, whereas $(i i)$ follows from $\left\langle E_{I}, e_{l}\right\rangle$ $(V, 0) \equiv 0$. For $(i i i)$, first observe that $\nabla_{e_{l}} e_{m}(V, 0)=0$ implies that map $v \mapsto \Phi(V, v)$ is geodesic at $v=0$, i.e. given any vector $\eta \in\left(T_{\psi(V)} \Pi_{V}\right)^{\perp}$,

$$
<\nabla_{\eta} e_{m}, e_{l}>(V, 0)=-<\nabla_{e_{m}} e_{l}, \eta>(V, 0)=0 .
$$

Thus,

$$
\begin{aligned}
e_{m}<E_{I}, e_{l}>(V, 0)= & <\nabla_{e_{m}} E_{I}, e_{l}>(V, 0)+<E_{I}, \nabla_{e_{m}} e_{l}>(V, 0) \\
= & <\nabla_{E_{I}} e_{m}, e_{l}>(V, 0)=0 .
\end{aligned}
$$

(iv) follows from the fact that $\psi\left(U_{0}\right)$ is a totally geodesic in $M$ and $(v)$ follows from $<e_{l}, e_{m}>(V, 0) \equiv \delta_{l m}$. Finally, $(v i)$ follows from $\nabla_{e_{l}} e_{m}(V, 0) \equiv 0$. Q.E.D.

If $x \in \mathcal{R}(u)$, then there exists $\delta>0$ and a DM $M$ such that $u\left(B_{\delta}\left(x_{0}\right)\right) \subset$ $M$. We apply Lemma 18 to obtain a coordinates $(V, v) \in \mathbf{R}^{j} \times \mathbf{R}^{k-j}$ with $G$ at $(V, v)$ represented by the matrix

$$
\left(\begin{array}{ll}
G_{11}(V, v) & G_{12}(V, v) \\
G_{21}(V, v) & G_{22}(V, v)
\end{array}\right) .
$$


Using these coordinates, interpret $\frac{\partial V}{\partial x^{\alpha}}$ as a vector in $\mathbf{R}^{j}$ and $\frac{\partial v}{\partial x^{\alpha}}$ and $\frac{\partial v_{\eta}}{\partial x^{\alpha}}$ as vectors in $\mathbf{R}^{k-j}$. For any $j \times j$-matrix $\mathcal{M}_{11}, j \times(k-j)$-matrix $\mathcal{M}_{12}$ and $(k-j) \times(k-j)$ matrix $\mathcal{M}_{22}$, we write

$$
\begin{gathered}
\mathcal{M}_{11} \nabla V \cdot \nabla V, \mathcal{M}_{12} \nabla V \cdot \nabla v, \mathcal{M}_{22} \nabla v \cdot \nabla v, \\
\mathcal{M}_{12} \nabla V \cdot \nabla v_{\eta}, \mathcal{M}_{22} \nabla v_{\eta} \cdot \nabla v_{\eta}
\end{gathered}
$$

to denote the inner products defined by

$$
\begin{array}{lc}
\sum_{\alpha, \beta=1}^{n} g^{\alpha \beta}\left(\frac{\partial V}{\partial x^{\alpha}}\right)^{T} \mathcal{M}_{11}\left(\frac{\partial V}{\partial x^{\beta}}\right) & \sum_{\alpha, \beta=1}^{n} g^{\alpha \beta}\left(\frac{\partial v}{\partial x^{\alpha}}\right)^{T} \mathcal{M}_{12}\left(\frac{\partial V}{\partial x^{\beta}}\right) \\
\sum_{\alpha, \beta=1}^{n} g^{\alpha \beta}\left(\frac{\partial v}{\partial x^{\alpha}}\right)^{T} \mathcal{M}_{22}\left(\frac{\partial v}{\partial x^{\beta}}\right) & \sum_{\alpha, \beta=1}^{n} g^{\alpha \beta}\left(\frac{\partial v_{\eta}}{\partial x^{\alpha}}\right)^{T} \mathcal{M}_{12}\left(\frac{\partial V}{\partial x^{\beta}}\right) \\
\sum_{\alpha, \beta=1}^{n} g^{\alpha \beta}\left(\frac{\partial v_{\eta}}{\partial x^{\alpha}}\right)^{T} \mathcal{M}_{22}\left(\frac{\partial v_{\eta}}{\partial x^{\beta}}\right) &
\end{array}
$$

respectively.

On the other hand, using an isometric embedding

$$
\iota:\left(\mathbf{R}^{j} \times Y_{2}^{k-j}, G\right) \hookrightarrow \mathbf{R}^{N},
$$

we will set the following notation for any fixed $x_{1} \in B_{\sigma}\left(x_{0}\right)$ :

$$
\begin{aligned}
G_{11}(V, v) \nabla V \cdot \nabla V & =\sum_{\alpha \beta} g^{\alpha \beta}<\frac{\partial\left(\iota \circ\left(V, v\left(x_{1}\right)\right)\right)}{\partial x^{\alpha}}, \frac{\partial\left(\iota \circ\left(V, v\left(x_{1}\right)\right)\right)}{\partial x^{\beta}}> \\
G_{12}(V, v) \nabla V \cdot \nabla v & =\sum_{\alpha \beta} g^{\alpha \beta}<\frac{\partial\left(\iota \circ\left(V, v\left(x_{1}\right)\right)\right)}{\partial x^{\alpha}}, \frac{\partial\left(\iota \circ\left(V\left(x_{1}\right), v\right)\right)}{\partial x^{\beta}}> \\
G_{22}(V, v) \nabla v \cdot \nabla v & =\sum_{\alpha \beta} g^{\alpha \beta}<\frac{\partial\left(\iota \circ\left(V\left(x_{1}\right), v\right)\right)}{\partial x^{\alpha}}, \frac{\partial\left(\iota \circ\left(V\left(x_{1}\right), v\right)\right)}{\partial x^{\beta}}> \\
G_{12}\left(V, v_{\eta}\right) \nabla V \cdot \nabla v_{\eta} & =\sum_{\alpha \beta} g^{\alpha \beta}<\frac{\partial\left(\iota \circ\left(V, v_{\eta}\left(x_{1}\right)\right)\right)}{\partial x^{\alpha}}, \frac{\partial\left(\iota \circ\left(V\left(x_{1}\right), v_{\eta}\right)\right)}{\partial x^{\beta}}> \\
G_{22}\left(V, v_{\eta}\right) \nabla v_{\eta} \cdot \nabla v_{\eta} & =\sum_{\alpha \beta} g^{\alpha \beta}<\frac{\partial\left(\iota \circ\left(V\left(x_{1}\right), v_{\eta}\right)\right)}{\partial x^{\alpha}}, \frac{\partial\left(\iota \circ\left(V\left(x_{1}\right), v_{\eta}\right)\right)}{\partial x^{\beta}}>.
\end{aligned}
$$

We note that for $x \in \mathcal{R}(u)$, the notation above is consistent with the notation of (21) replacing $\mathcal{M}_{11}, \mathcal{M}_{12}$ and $\mathcal{M}_{22}$ by $G_{11}(V, v), G_{12}(V, v)$ and $G_{22}(V, v)$ of (20). Same statement for (22) is true with $\mathcal{M}_{12}$ and $\mathcal{M}_{22}$ by replaced by $G_{12}\left(V, v_{\eta}\right)$ and $G_{22}\left(V, v_{\eta}\right)$ of $(20)$. 


\section{Target Variation}

In this section, we obtain estimates for the singular component map $v$ of a harmonic map $u=(V, v): B_{\sigma_{\star}}\left(x_{\star}\right) \rightarrow\left(\mathbf{R}^{j} \times Y_{2}{ }^{k-j}, G\right)$. We start with the following

Definition 19 We say a neighborhood $N$ of $\left(0, P_{0}\right)$ is good if for any DM $M$ of $\left(\mathbf{R}^{j} \times Y_{2}^{k-j}, G\right)$, there exist coordinates $\Phi: \mathcal{U} \subset \mathbf{R}^{j} \times \mathbf{R}^{k-j} \rightarrow M$ as in Lemma 18 such that $N \subset \Phi(\mathcal{U})$.

Note that by choosing $\sigma_{\star}>0$ sufficiently small, we can assure that $u\left(B_{\sigma_{\star}}\left(x_{\star}\right)\right)$ is contained in a good neighborhood of $u\left(x_{\star}\right)$. We will make the following assumptions for a harmonic map $u$.

Assumption 20 (i) The image $u\left(B_{\sigma_{\star}}\left(x_{\star}\right)\right)$ is contained in a good neighborhood of $u\left(x_{\star}\right)=\left(0, P_{0}\right)$.

(ii) The metric $G\left(u\left(x_{\star}\right)\right)=H \times h$ on $\mathbf{R}^{j} \times Y_{2}^{k-2}$ defines an F-connected complex.

(iii) The set of singular points of $u$ not in $\mathcal{S}_{j}(u)$ is of Hausdorff codimension at least 2, i.e.

$$
\operatorname{dim}_{\mathcal{H}} \mathcal{S}_{j+1}^{+}(u) \leq n-2 .
$$

We now proceed with analyzing $u$ satisfying the assumptions above.

Proposition 21 Let $u=(V, v): B_{\sigma_{\star}}\left(x_{\star}\right) \rightarrow\left(\mathbf{R}^{j} \times Y_{2}{ }^{k-j}, G\right)$ satisfy Assumption 20. For $x_{0} \in \mathcal{S}_{j}(u) \cap B_{\frac{\sigma_{\star}}{2}}\left(x_{\star}\right)$ and $\sigma>0$ such that $B_{\sigma}\left(x_{0}\right) \subset B_{\sigma_{\star}}\left(x_{\star}\right)$, let $\eta \in C_{c}^{\infty}\left(B_{\sigma}\left(x_{0}\right)\right)$ with $0 \leq \eta \leq 1$ and $v_{\eta}: B_{\sigma}\left(x_{0}\right) \rightarrow Y_{2}^{k-j}$ be as in Lemma 17 . Then

$$
E_{x_{0}}^{v}(\sigma)-E_{x_{0}}^{v_{\eta}}(\sigma) \leq C \int_{B_{\sigma}\left(x_{0}\right)} \eta d^{2}\left(v, P_{0}\right) d \mu .
$$

Furthermore, the constant $C$ depends only on the $C^{2}$ norms of the metrics $g$ and $G$ and the Lipschitz constant of $u$.

Proof. Since $u$ is harmonic, we have under the notation as in Lemma 17

$$
0 \geq E_{x_{0}}^{u}(\sigma)-E_{x_{0}}^{u_{\eta}}(\sigma)
$$




$$
\begin{aligned}
= & \int_{B_{\sigma}\left(x_{0}\right)}|\nabla u|^{2} d \mu-\int_{B_{\sigma}\left(x_{0}\right)}\left|\nabla u_{\eta}\right|^{2} d \mu \\
= & \int_{B_{\sigma}\left(x_{0}\right)}\left(G_{11}(V, v) \nabla V \cdot \nabla V-G_{11}\left(V, v_{\eta}\right) \nabla V \cdot \nabla V\right) d \mu \\
& +2 \int_{B_{\sigma}\left(x_{0}\right)}\left(G_{12}(V, v) \nabla V \cdot \nabla v-G_{12}\left(V, v_{\eta}\right) \nabla V \cdot \nabla v_{\eta}\right) d \mu \\
& +\int_{B_{\sigma}\left(x_{0}\right)}\left(G_{22}(V, v) \nabla v \cdot \nabla v-G_{22}\left(V, v_{\eta}\right) \nabla v_{\eta} \cdot \nabla v_{\eta}\right) d \mu
\end{aligned}
$$

where we have used the notation introduced at the end of Section 4 for the last inequality. By Lemma 17, $|\nabla u|^{2}=\left|\nabla u_{\eta}\right|^{2}$ for a.e $x \in \mathcal{S}_{j}(u)$. Thus we can rewrite this inequality as

$$
\begin{aligned}
\int_{B_{\sigma}\left(x_{0}\right) \backslash \mathcal{S}_{j}(u)}\left(G_{22}(V, v) \nabla v \cdot \nabla v-G_{22}\left(V, v_{\eta}\right) \nabla v_{\eta} \cdot \nabla v_{\eta}\right) d \mu \\
\leq \quad \int_{B_{\sigma}\left(x_{0}\right) \backslash \mathcal{S}_{j}(u)}\left(G_{11}\left(V, v_{\eta}\right) \nabla V \cdot \nabla V-G_{11}(V, v) \nabla V \cdot \nabla V\right) d \mu \\
\quad+2 \int_{B_{\sigma}\left(x_{0}\right) \backslash \mathcal{S}_{j}(u)}\left(G_{12}\left(V, v_{\eta}\right) \nabla V \cdot \nabla v_{\eta}-G_{12}(V, v) \nabla V \cdot \nabla v\right) d \mu \\
=: \quad(I)+(I I) .
\end{aligned}
$$

We will first prove the estimate

$$
(I)+(I I) \leq C \int_{B_{\sigma}\left(x_{0}\right)} \eta d_{p_{0}}^{2} d \mu
$$

where $C>0$ above and in the rest of the proof is a constant that depends only on the $C^{2}$ norms of the metrics $g$ and $G$ and the Lipschitz constant of $u$. To prove (25), we will use the fact that $V$ and $v$ are Lipschitz, Lemma 18 on the estimates of the metric and $d\left(v_{\eta}(x), v(x)\right)=\eta d_{P_{0}}(x)$.

We first estimate $(I)$. Let $x \in B_{\sigma}\left(x_{0}\right) \cap \mathcal{R}(u)$ and let $M$ be a DM containing $u\left(B_{\delta}(x)\right)$ for some $\delta>0$. Using the coordinates of Lemma 18 and the Mean Value Theorem, we can write at $x$

$$
\begin{aligned}
\left|\left(G_{11}\left(V, v_{\eta}\right)-G_{11}(V, v)\right) \nabla V \cdot \nabla V\right| & =\left|\frac{\partial}{\partial v} G_{11}(V, \xi)\right| \eta d_{P_{0}}|\nabla V \cdot \nabla V| \\
& \leq C|\xi| \eta d_{P_{0}} \\
& \leq C \eta d_{P_{0}}^{2} .
\end{aligned}
$$


Since $\operatorname{dim}_{\mathcal{H}}\left(\mathcal{S}_{j+1}^{+}(u)\right) \leq n-2$, this proves

$$
(I):=\int_{B_{\sigma}\left(x_{0}\right) \backslash \mathcal{S}_{j}(u)}\left(G_{11}\left(V, v_{\eta}\right) \nabla V \cdot \nabla V-G_{11}(V, v) \nabla V \cdot \nabla V\right) d \mu \leq C \eta d_{P_{0}}^{2} .
$$

To estimate $(I I)$, define

$$
A_{\epsilon}^{+}=\left\{x \in \overline{B_{\sigma}\left(x_{0}\right)}:|v|>\epsilon\right\} .
$$

We claim that

$$
\exists \epsilon_{j} \rightarrow 0 \text { such that } \epsilon_{j} \mathcal{H}^{n-1}\left(\partial A_{\epsilon_{j}}^{+} \cap B_{\sigma}\left(x_{0}\right)\right) \rightarrow 0 .
$$

If $\epsilon \mathcal{H}^{n-1}\left(\partial A_{\epsilon}^{+} \cap B_{\sigma}\left(x_{0}\right)\right) \geq \delta>0$ for $\epsilon<\epsilon_{0}$, then we have

$$
\int_{0}^{\epsilon_{0}} \mathcal{H}^{n-1}\left(\partial A_{\epsilon}^{+} \cap B_{\sigma}\left(x_{0}\right)\right) d \epsilon \geq \delta \int_{0}^{\epsilon_{0}} \frac{1}{\epsilon} d \epsilon=\infty ;
$$

on the other hand, the co-area formula and the fact that $|v|$ is Lipschitz imply that

$$
\int_{0}^{\infty} \mathcal{H}^{n-1}\left(\partial A_{\epsilon}^{+} \cap B_{\sigma}\left(x_{0}\right)\right) d \epsilon=\int_{A_{0}^{+}}|\nabla| v|| d \mu=\int_{B_{\sigma}\left(x_{0}\right)}|\nabla| v|| d \mu<\infty,
$$

which is a contradiction and this proves (28).

For $x \in\left(B_{\sigma}\left(x_{0}\right) \backslash A_{\epsilon_{j}}^{+}\right) \cap \mathcal{R}(u)$, let $M$ be a DM containing $u\left(B_{\delta}(x)\right)$ for some $\delta>0$ and $P_{0}$. Using the coordinates of Lemma 18, at $x$ we have

$$
\left|G_{12}(V, v) \nabla V \cdot \nabla v\right|,\left|G_{12}\left(V, v_{\eta}\right) \nabla V \cdot \nabla v_{\eta}\right| \leq C|v|^{2} .
$$

Since $\mathcal{H}^{n}\left(\mathcal{S}_{j+1}^{+}\right)=0$, this implies

$$
\begin{aligned}
(I I) & :=2 \int_{B_{\sigma}\left(x_{0}\right) \backslash \mathcal{S}_{j}(u)}\left(G_{12}\left(V, v_{\eta}\right) \nabla V \cdot \nabla v_{\eta}-G_{12}(V, v) \nabla V \cdot \nabla v\right) d \mu \\
& =2 \int_{A_{\epsilon_{j}}^{+}}\left(G_{12}\left(V, v_{\eta}\right) \nabla V \cdot \nabla v_{\eta}-G_{12}(V, v) \nabla V \cdot \nabla v\right) d \mu+O\left(\epsilon_{j}^{2}\right) .
\end{aligned}
$$

We can now write

$$
\begin{aligned}
(I I)= & 2 \int_{A_{\epsilon_{j}}^{+}}\left(G_{12}\left(V, v_{\eta}\right)-G_{12}(V, v)\right) \nabla V \cdot \nabla v_{\eta} d \mu \\
& +2 \int_{A_{\epsilon_{j}}^{+}} G_{12}(V, v)\left(\nabla V \cdot \nabla v_{\eta}-\nabla V \cdot \nabla v\right) d \mu+O\left(\epsilon_{j}^{2}\right) \\
=: & (I I)_{1}+(I I)_{2}+O\left(\epsilon_{j}^{2}\right) .
\end{aligned}
$$


We estimate $(I I)_{1}$ in similar way as $(I)$. Next, we prove the corresponding inequality for $(I I)_{2}$. We first justify the following formula

$$
\begin{aligned}
\int_{A_{\epsilon}^{+}} & G_{12}(V, v)_{I i}<\nabla V^{I}, \nabla v^{i}>d \mu-\int_{A_{\epsilon}^{+}} G_{12}\left(V, v_{\eta}\right)_{I i}<\nabla V^{I}, \nabla v_{\eta}^{i}>d \mu \\
= & -\int_{A_{\epsilon}^{+}} \operatorname{div}\left(G_{12}(V, v)_{I i} \nabla V^{I}\right) v^{i} d \mu+\int_{A_{\epsilon}^{+}} \operatorname{div}\left(G_{12}\left(V, v_{\eta}\right)_{I i} \nabla V^{I}\right) v_{\eta}^{i} d \mu \\
& +O\left(\epsilon_{j}^{2}\right)
\end{aligned}
$$

where $\langle\cdot, \cdot\rangle$ is the inner product with respect to the domain metric. To see this, let $\varrho>0$ and $\left\{B_{r_{l}}\left(x_{l}\right): l=1,2, \ldots\right\}$ be a cover of the set $\mathcal{S}_{j+1}^{+}(u)$ such that $\sum_{l=1}^{\infty} r_{j}^{n-1}<\varrho$. Let $\varphi_{\varrho}$ be a Lipschitz cut-off function which is zero in $\cup_{l=1}^{\infty} B_{r_{l}}\left(x_{l}\right)$ and identically one in $\Omega \backslash \cup_{l=1}^{\infty} B_{2 r_{l}}\left(x_{l}\right)$ with $\left|\nabla \varphi_{\varrho}\right| \leq 2 r_{l}^{-1}$ in $B_{r_{l}}\left(x_{l}\right)$. The divergence theorem implies

$$
\begin{aligned}
& \int_{A_{\epsilon_{j}}^{+}} \varphi_{\varrho} G_{12}(V, v)_{I i}<\nabla V^{I}, \nabla v^{i}>d \mu \\
& \quad=-\int_{A_{\epsilon_{j}}^{+}} \varphi_{\varrho} \operatorname{div}\left(G_{12}(V, v)_{I i} \nabla V^{I}\right) v^{i} d \mu-\int_{A_{\epsilon_{j}}^{+}} G_{12}(V, v)_{I i}<\nabla \varphi_{\varrho}, \nabla V^{I}>v^{i} d \mu \\
& \quad+\int_{\partial A_{\epsilon_{j}}^{+}} \varphi_{\varrho} G_{12}(V, v)_{I i} \frac{\partial V^{I}}{\partial r} v^{i} d \Sigma
\end{aligned}
$$

Similarly,

$$
\begin{aligned}
& \int_{A_{\epsilon_{j}}^{+}} \varphi_{\varrho} G_{12}\left(V, v_{\eta}\right)_{I i}<\nabla V^{I}, \nabla v_{\eta}^{i}>d \mu \\
& \quad=-\int_{A_{\epsilon_{j}}^{+}} \varphi_{\varrho} \operatorname{div}\left(G_{12}\left(V, v_{\eta}\right)_{I i} \nabla V^{I}\right) v_{\eta}^{i} d \mu-\int_{A_{\epsilon_{j}}^{+}} G_{12}\left(V, v_{\eta}\right)_{I i}<\nabla \varphi_{\varrho}, \nabla V^{I}>v_{\eta}^{i} d \mu \\
& \quad+\int_{\partial A_{\epsilon_{j}}^{+}} \varphi_{\varrho} G_{12}\left(V, v_{\eta}\right)_{I i} \frac{\partial V^{I}}{\partial r} v_{\eta}^{i} d \Sigma .
\end{aligned}
$$

For $x \in A_{\epsilon}^{+} \cap \mathcal{R}(u)$, let $M$ be the DM such that $u\left(B_{\delta}(x) \subset M\right.$ for $\delta>0$. Then using coordinates of Lemma 18, we have the estimate $\left|G_{12}(V, v)\right|<C|v|^{2}$. Thus, using (28), we conclude

$$
\left|\int_{\partial A_{\epsilon_{j}}^{+} \cap B_{\sigma}\left(x_{0}\right)} \varphi_{\varrho} G_{12}(V, v)_{I i} \frac{\partial V^{I}}{\partial r} v^{i} d \Sigma\right| \leq C \epsilon_{j}^{3} \mathcal{H}^{n-1}\left(\partial A_{\epsilon_{j}}^{+} \cap B_{\sigma}\left(x_{0}\right)\right)=O\left(\epsilon_{j}^{2}\right) .
$$


Thus, we have

$$
\begin{aligned}
& \int_{\partial A_{\epsilon_{j}}^{+}} \varphi_{\varrho} G_{12}(V, v)_{I i} \frac{\partial V^{I}}{\partial r} v^{i} d \Sigma \\
& =\int_{A_{\epsilon_{j}}^{+} \cap \partial B_{\sigma}\left(x_{0}\right)}+\int_{\partial A_{\epsilon_{j}}^{+} \cap B_{\sigma}\left(x_{0}\right)} \varphi_{\varrho} G_{12}(V, v)_{I i} \frac{\partial V^{I}}{\partial r} v^{i} d \Sigma \\
& =\int_{A_{\epsilon_{j}}^{+} \cap \partial B_{\sigma}\left(x_{0}\right)} \varphi_{\varrho} G_{12}(V, v)_{I i} \frac{\partial V^{I}}{\partial r} v^{i} d \Sigma+O\left(\epsilon_{j}^{2}\right) .
\end{aligned}
$$

Similarly,

$$
\begin{aligned}
& \int_{\partial A_{\epsilon_{j}}^{+}} \varphi_{\varrho} G_{12}\left(V, v_{\eta}\right)_{I i} \frac{\partial V^{I}}{\partial r} v_{\eta}^{i} d \Sigma \\
& \quad=\int_{A_{\epsilon_{j}}^{+} \cap \partial B_{\sigma}\left(x_{0}\right)} \varphi_{\varrho} G_{12}\left(V, v_{\eta}\right)_{I i} \frac{\partial V^{I}}{\partial r} v_{\eta}^{i} d \Sigma+O\left(\epsilon_{j}^{2}\right) .
\end{aligned}
$$

Additionally, note that since $v=v_{\eta}$ on $\partial B_{\sigma}\left(x_{0}\right)$,

$$
\int_{A_{\epsilon_{j}}^{+} \cap \partial B_{\sigma}\left(x_{0}\right)} \varphi_{\varrho} G_{12}(V, v)_{I i} \frac{\partial V^{I}}{\partial r} v^{i} d \Sigma=\int_{A_{\epsilon_{j}}^{+} \cap \partial B_{\sigma}\left(x_{0}\right)} \varphi_{\varrho} G_{12}\left(V, v_{\eta}\right)_{I i} \frac{\partial V^{I}}{\partial r} v_{\eta}^{i} d \Sigma .
$$

Furthermore,

$$
\begin{aligned}
\int_{A_{\epsilon_{j}}^{+}} G_{12}(V, v)_{I i}<\nabla \varphi_{\varrho}, \nabla V^{I}>v^{i} d \mu & \leq C \sum_{l=1}^{L} \frac{1}{r_{l}} \operatorname{Vol}\left(B_{r_{l}}\left(x_{l}\right)\right) \\
& \leq C \sum_{l=1}^{L} r_{l}^{n-1}=O(\varrho)
\end{aligned}
$$

and

$$
\begin{aligned}
\int_{A_{\epsilon_{j}}^{+}} G_{12}\left(V, v_{\eta}\right)_{I i}<\nabla \varphi_{\varrho}, \nabla V^{I}>v_{\eta}^{i} d \mu & \leq C \sum_{l=1}^{L} \frac{1}{r_{l}} \operatorname{Vol}\left(B_{r_{l}}\left(x_{l}\right)\right) \\
& \leq C \sum_{l=1}^{L} r_{l}^{n-1}=O(\varrho) .
\end{aligned}
$$

Subtracting (32) from (31) and using (33), (34), (35), (36) and (37) and letting $\varrho \rightarrow 0$, we have justified (30). 
We use (30) to write

$$
\begin{aligned}
(I I)_{2} & =2 \int_{A_{\epsilon_{j}}^{+}} G_{12}\left(V, v_{\eta}\right)_{I i}<\nabla V^{I}, \nabla v_{\eta}^{i}>-G_{12}\left(V, v_{\eta}\right)_{I i}<\nabla V^{I}, \nabla v^{i}>d \mu \\
& =-\int_{A_{\epsilon_{j}}^{+}} \operatorname{div}\left(G_{12}(V, v)_{I i} \nabla V^{I}\right) v^{i} d \mu+\int_{A_{\epsilon_{j}}^{+}} \operatorname{div}\left(G_{12}(V, v)_{I i} \nabla V^{I}\right) v^{i} d \mu+O\left(\epsilon_{j}^{2}\right) \\
& =2((A)+(B))+O\left(\epsilon_{j}^{2}\right)
\end{aligned}
$$

where $(A)$ and $(B)$ are defined below. Again using the coordinates of Lemma 18, we have $\left|\dot{G}_{12}(V, v)\right|<C|v|$ which implies that

$$
\begin{aligned}
(A) & :=\int_{A_{\epsilon_{j}^{+}}^{+}}\left(<\nabla G_{12}(V, v)_{I i}, \nabla V^{I}>v_{\eta}^{i}-<\nabla G_{12}(V, v)_{I i}, \nabla V^{I}>v^{i}\right) d \mu \\
& \leq C \int_{B_{\sigma}\left(x_{0}\right)}|v| \eta d_{P_{0}} d \mu=C \int_{B_{\sigma}\left(x_{0}\right)} \eta d_{P_{0}}^{2} d \mu .
\end{aligned}
$$

Next, using again the estimate $\left|G_{12}(V, v)\right|<C|v|^{2}$ and the harmonic map equation to bound $\triangle V$, we have

$$
\begin{aligned}
(B) & :=\int_{A_{\epsilon_{j}^{+}}^{+}}\left(G_{12}\left(V, v_{I i} \triangle V^{I} v_{\eta}^{i}-G_{12}(V, v)_{I i} \triangle V^{I} v^{i}\right) d \mu\right. \\
& \leq C \int_{B_{\sigma}\left(x_{0}\right)}|v|^{2} \eta d_{P_{0}} d \mu=C \int_{B_{\sigma}\left(x_{0}\right)} \eta d_{P_{0}}^{2} d \mu
\end{aligned}
$$

Therefore,

$$
\begin{aligned}
(I I)_{2} & =2((A)+(B))+O\left(\epsilon_{j}^{2}\right) \\
& \leq C \int_{B_{\sigma}\left(x_{0}\right)} \eta d_{p_{0}}^{2} d \mu+O\left(\epsilon_{j}^{2}\right)
\end{aligned}
$$

Thus, we have shown

$$
(I I)=(I I)_{1}+(I I)_{2}+O\left(\epsilon_{j}^{2}\right) \leq C \int_{B_{\sigma}\left(x_{0}\right)} \eta d_{p_{0}}^{2} d \mu+O\left(\epsilon_{j}^{2}\right) .
$$

Letting $\epsilon_{j} \rightarrow 0$, we have completed the proof of the estimate (25). Combining (24) and (25), we have shown that

$$
\int_{B_{\sigma}\left(x_{0}\right) \backslash \mathcal{S}_{j}(u)}\left(G_{22}(V, v) \nabla v \cdot \nabla v-G_{22}\left(V, v_{\eta}\right) \nabla v_{\eta} \cdot \nabla v_{\eta}\right) d \mu \leq C \int_{B_{\sigma}\left(x_{0}\right)} \eta d_{P_{0}}^{2} d \mu .
$$


Finally, we claim

$$
\begin{aligned}
(I I I) & :=\mid \int_{B_{\sigma}\left(x_{0}\right) \backslash \mathcal{S}_{j}(u)}\left(|\nabla v|^{2}-\left|\nabla v_{\eta}\right|^{2}\right) d \mu \\
& -\int_{B_{\sigma}\left(x_{0}\right) \backslash \mathcal{S}_{j}(u)}\left(G_{22}(V, v) \nabla v \cdot \nabla v-G_{22}\left(V, v_{\eta}\right) \nabla v_{\eta} \cdot \nabla v_{\eta}\right) d \mu \mid \\
\leq & C \int_{B_{\sigma}\left(x_{0}\right)} \eta d_{P_{0}}^{2} d \mu
\end{aligned}
$$

Notice that (39) completes the proof of the Proposition. Indeed, since $|\nabla v|^{2}=\left|\nabla v_{\eta}\right|^{2}$ a.e on $\mathcal{S}_{j}(u)$ by Lemma 17, we have

$$
h_{x_{0}} E^{v}(\sigma)-h_{x_{0}} E^{v}(\sigma)=\int_{B_{\sigma}\left(x_{0}\right) \backslash \mathcal{S}_{j}(u)}\left(|\nabla v|^{2}-\left|\nabla v_{\eta}\right|^{2}\right) d \mu
$$

and combining the above with (38) and (39) proves (23) and finishes the proof.

We now proceed with the proof of (39). For each DM $M$ of $\mathbf{R}^{j} \times Y_{2}^{k-j}$, consider the coordinates $(V, v)$ of $M$ given in Lemma 18. By (18), the metric $H \times h$ restricted to $M$ with respect to this coordinate system is given by the identity matrix $I$. In particular, this means we can write

$$
|\nabla v|^{2}=I \nabla v \cdot \nabla v \text { and }\left|\nabla v_{\eta}\right|^{2}=I \nabla v_{\eta} \cdot \nabla v_{\eta}
$$

in $B_{\delta}\left(x_{0}\right)$. Here, note that we are using the notation introduced in (21) and $(22)$.

Let $x \in B_{\sigma}\left(x_{0}\right) \cap \mathcal{R}(u)$ and let $M$ be the DM containing $u\left(B_{\delta}(x)\right)$ for some $\delta>0$. Using the coordinates of Lemma 18, we have $G_{22}(V, 0)=I$ for all $V$ and the estimates

$$
\left|\square_{0}\right| \leq C|v|^{2} \text { and }\left|\square_{\eta}\right| \leq C\left|v_{\eta}\right|^{2} \leq C|v|^{2},
$$

where

$$
\square_{0}=I-G_{22}(V, v) \text { and } \square_{\eta}=I-G_{22}\left(V, v_{\eta}\right) .
$$

Therefore, using (40), we obtain

$$
\left.|| \nabla v\right|^{2}-\left.G_{22}(V, v) \nabla v \cdot \nabla v|=| \square_{0} \nabla v \cdot \nabla v|\leq C| v\right|^{2}
$$

and

$$
\left.|| \nabla v_{\eta}\right|^{2}-\left.G_{22}\left(V, v_{\eta}\right) \nabla v_{\eta} \cdot \nabla v_{\eta}|=| \square_{\eta} \nabla v_{\eta} \cdot \nabla v_{\eta}|\leq C| v\right|^{2}
$$


for $x \in B_{\sigma}\left(x_{0}\right) \cap \mathcal{R}(u)$. Since $\operatorname{dim}_{\mathcal{H}}\left(\mathcal{S}_{j+1}^{+}(u)\right) \leq n-2$, we have

$$
(I I I)=\left|\int_{A_{\epsilon}^{+}}\left(\square_{0} \nabla v \cdot \nabla v d \mu-\square_{\eta} \nabla v_{\eta} \cdot \nabla v_{\eta}\right) d \mu\right|+O\left(\epsilon^{2}\right)
$$

and we can write

$$
\begin{aligned}
(I I I) \leq & \left|\int_{A_{\epsilon}^{+}}\left(\square_{0}-\square_{\eta}\right) \nabla v \cdot \nabla v d \mu\right|+\left|\int_{A_{\epsilon}^{+}} \square_{\eta}\left(\nabla v \cdot \nabla v-\nabla v \cdot \nabla v_{\eta}\right) d \mu\right| \\
& +\left|\int_{A_{\epsilon}^{+}} \square_{\eta}\left(\nabla v \cdot \nabla v_{\eta}-\nabla v_{\eta} \cdot \nabla v_{\eta}\right) d \mu\right|+O\left(\epsilon^{2}\right) \\
= & (a)+(b)+(c)+O\left(\epsilon^{2}\right) .
\end{aligned}
$$

For $x \in A_{\epsilon}^{+} \cap \mathcal{R}(u)$, we have by the mean value theorem exactly as in (26)

$$
\begin{aligned}
\left|\square_{0} \nabla v \cdot \nabla v-\square_{\eta} \nabla v \cdot \nabla v\right| & =\left|\left(G_{22}(V, v)-G_{22}\left(V, v_{\eta}\right)\right) \nabla v \cdot \nabla v\right| \\
& \leq C|v| \eta d_{P_{0}} .
\end{aligned}
$$

Hence

$$
(a) \leq C \int_{A_{\epsilon}^{+}} \eta d_{P_{0}}^{2} d \mu
$$

Next, we have the estimates $\left|\nabla \square_{\eta}\right| \leq C|v|$ and $\left|\square_{\eta}\right| \leq C|v|^{2}$, hence we can use the divergence theorem (which is justified in the same way as previously) to write

$$
\begin{aligned}
(b) & =\left|\int_{A_{\epsilon}^{+}} \operatorname{div}\left(\square_{\eta} \nabla v\right)\left(v-v_{\eta}\right) d \mu\right| \\
& \leq\left|\int_{A_{\epsilon}^{+}} \nabla \square_{\eta} \cdot \nabla v\left(v-v_{\eta}\right) d \mu\right|+\left|\int_{A_{\epsilon}^{+}} \square_{\eta} \triangle v\left(v-v_{\eta}\right) d \mu\right| \\
& \leq C \int_{A_{\epsilon}^{+}} \eta d_{P_{0}}^{2} d \mu .
\end{aligned}
$$

Finally, $(c)$ can be estimated exactly as $(b)$. We let $\epsilon \rightarrow 0$ and we obtain (39). Q.E.D.

Remark. Notice that the proof of the previous proposition is significantly complicated by the fact that we need to estimate the right hand side in terms of $\eta d_{P_{0}}^{2}$. If we were willing to replace $\eta$ by 1 , which is the case in the following Proposition, then for example the application of the divergence theorem would be unnecessary. 
Proposition 22 Let $u=(V, v): B_{\sigma_{\star}}\left(x_{\star}\right) \rightarrow\left(\mathbf{R}^{j} \times Y_{2}{ }^{k-j}, G\right)$ satisfy Assumption 20. For $x_{0} \in \mathcal{S}_{j}(u) \cap B_{\frac{\sigma_{\star}}{2}}\left(x_{\star}\right)$ and $\sigma>0$ such that $B_{\sigma}\left(x_{0}\right) \subset B_{\sigma_{\star}}\left(x_{\star}\right)$, let $w: B_{\sigma}\left(x_{0}\right) \rightarrow\left(Y_{2}^{k-j}, h\right)$ be a harmonic map with $\left.w\right|_{\partial B_{\sigma}\left(x_{0}\right)}=\left.v\right|_{\partial B_{\sigma}\left(x_{0}\right)}$. Then

$$
E_{x_{0}}^{v}(\sigma)-E_{x_{0}}^{w}(\sigma) \leq C \int_{B_{\sigma}\left(x_{0}\right)}\left(d^{2}\left(v, P_{0}\right)+d^{2}(w, v)\right) d \mu .
$$

Furthermore, the constant $C$ depends only on the $C^{2}$ norms of the metrics $g$ and $G$ and the Lipschitz constants of $u$ and $w$.

Proof. By [GS] Theorem 6.4, we have $\operatorname{dim}_{\mathcal{H}} \mathcal{S}(w) \leq n-2$. Noting that $|\nabla v|^{2}(x)=0$ for a.e. $x \in \mathcal{S}_{j}(u)$ by Lemma 17 , we can write

$$
E_{x_{0}}^{v}(\sigma)-E_{x_{0}}^{w}(\sigma) \leq \int_{B_{x_{0}}(\sigma) \backslash \mathcal{S}_{j}(u)}\left(|\nabla v|^{2}-|\nabla w|^{2}\right) d \mu .
$$

With this, we can follow the the proof of Proposition 21 with $w$ replacing $v_{\eta}$. The only difference here is that we do not use the equality $d\left(v_{\eta}(x), v(x)\right)=$ $\eta d_{P_{0}}(x)$. See also Remark preceding this Proposition. Therefore, we obtain $d^{2}(w, v)$ in the integral on the right hand side of (41). Q.E.D.

Next we will prove the following auxiliary

Lemma 23 Let $u=(V, v): B_{\sigma_{\star}}\left(x_{\star}\right) \rightarrow\left(\mathbf{R}^{j} \times Y_{2}^{k-j}, G\right)$ satisfy Assumption 20. For $x_{0} \in \mathcal{S}_{j}(u) \cap B \frac{\sigma_{\star}}{2}\left(x_{\star}\right)$ and $\sigma>0$ such that $B_{\sigma}\left(x_{0}\right) \subset B_{\sigma_{\star}}\left(x_{\star}\right)$, let $w: B_{\sigma}\left(x_{0}\right) \rightarrow\left(Y_{2}^{k-j}, h\right)$ be a harmonic map with $\left.w\right|_{\partial B_{\sigma}\left(x_{0}\right)}=\left.v\right|_{\partial B_{\sigma}\left(x_{0}\right)}$. Then,

$$
\int_{B_{\sigma}\left(x_{0}\right)} d^{2}\left(v, P_{0}\right)+d^{2}\left(w, P_{0}\right) d \mu \leq C\left(\sigma I_{x_{0}}^{v}(\sigma)+\sigma^{2} E_{x_{0}}^{v}(\sigma)\right) .
$$

Furthermore, the constant $C>0$ depends only on the $C^{2}$ norm of the metric $g$.

Proof. By the usual monotonicity formula for harmonic maps (cf. [GS], last formula on p. 195), there exists a constant $C>0$ depending on the $C^{2}$ norm of the metric $g$ such that

$$
\int_{B_{\sigma}\left(x_{0}\right)} d^{2}\left(w, P_{0}\right) d \mu \leq C \sigma \int_{\partial B_{\sigma}\left(x_{0}\right)} d^{2}\left(w, P_{0}\right) d \Sigma=C \sigma I_{x_{0}}^{v}(\sigma) .
$$


Let $w_{1 / 2}: B_{\sigma} \rightarrow Y_{2}$ be the map defined by setting $w_{1 / 2}(x)$ to be the midpoint of the geodesic between $v(x)$ and $w(x)$. Then by (2.2iv) of [KS2], we have

$$
2 E_{x_{0}}^{w_{1 / 2}} \leq E_{x_{0}}^{v}+E_{x_{0}}^{w}-\frac{1}{2} \int_{B_{\sigma}\left(x_{0}\right)}|\nabla d(v, w)|^{2} d \mu .
$$

The harmonicity of $w$ implies $E_{x_{0}}^{w} \leq E_{x_{0}}^{w_{1 / 2}}$ which in turn implies

$$
\int_{B_{\sigma}\left(x_{0}\right)}|\nabla d(v, w)|^{2} d \mu \leq E_{x_{0}}^{v}-E_{x_{0}}^{w_{1 / 2}} \leq E_{x_{0}}^{v} .
$$

By the Poincare Inequality, we obtain

$$
\int_{B_{\sigma}\left(x_{0}\right)} d^{2}(v, w) d \mu \leq C \sigma^{2} \int_{B_{\sigma}\left(x_{0}\right)}|\nabla d(v, w)|^{2} d \mu \leq C \sigma^{2} E_{x_{0}}^{v} .
$$

Combining (43) and (44) with the triangle inequality

$$
\int_{B_{\sigma}\left(x_{0}\right)} d^{2}\left(v, P_{0}\right) d \mu \leq 2 \int_{B_{\sigma}\left(x_{0}\right)} d^{2}\left(w, P_{0}\right) d \mu+2 \int_{B_{\sigma}\left(x_{0}\right)} d^{2}(v, w) d \mu
$$

completes the proof. Q.E.D.

The next proposition is the main result of the section. It is the analogue of the target variation formula (2.2) of [GS].

Proposition 24 Let $u=(V, v): B_{\sigma_{\star}}\left(x_{\star}\right) \rightarrow\left(\mathbf{R}^{j} \times Y_{2}{ }^{k-j}, G\right)$ satisfy Assumption 20. For $x_{0} \in \mathcal{S}_{j}(u) \cap B_{\frac{\sigma_{\star}}{2}}\left(x_{\star}\right)$ and $\sigma>0$ such that $B_{\sigma}\left(x_{0}\right) \subset B_{\sigma_{\star}}\left(x_{\star}\right)$, there exists $C>0$ such that

$$
2 E_{x_{0}}^{v}(\sigma) \leq \int_{\partial B_{\sigma}\left(x_{0}\right)} \frac{\partial}{\partial r} d^{2}\left(v, P_{0}\right) d \mu+C\left(\sigma I_{x_{0}}^{v}(\sigma)+\sigma^{2} E_{x_{0}}^{v}(\sigma)\right) .
$$

Furthermore the constant $C$ depends only on the $C^{2}$ norms of the metrics $g$ and $G$ and the Lipschitz constant of $u$.

Proof. For a non-negative smooth function $\eta \in C_{c}^{\infty}\left(B_{\sigma}\left(x_{0}\right)\right)$ with $0 \leq$ $\eta \leq 1$ and $t$ sufficiently small, let $v_{t \eta}: \Omega \rightarrow Y_{2}$ as in Lemma 17 with $\eta$ replaced by $t \eta$. From [GS] Section 2,

$$
E_{x_{0}}^{v_{t \eta}}(\sigma) \leq \int_{B_{\sigma}\left(x_{0}\right)}(1-t \eta)^{2}|\nabla v|^{2} d \mu-t \int_{B_{\sigma}\left(x_{0}\right)} \nabla \eta \cdot \nabla d^{2}\left(v_{t \eta}(x), P_{0}\right) d \mu+0\left(t^{2}\right) .
$$


Hence rearranging terms, dividing by $t$ and letting $t \rightarrow 0$, we obtain

$$
\begin{aligned}
& 2 \int_{B_{\sigma}\left(x_{0}\right)} \eta|\nabla v|^{2} d \mu \\
& \quad \leq-\int_{B_{\sigma}\left(x_{0}\right)} \nabla \eta \cdot \nabla d^{2}\left(v(x), P_{0}\right) d \mu+\liminf _{t \rightarrow 0} \frac{E_{x_{0}}^{v}(\sigma)-E_{x_{0}}^{v_{t \eta}}(\sigma)}{t} .
\end{aligned}
$$

Replacing $\eta$ by $t \eta$ in Proposition 21, dividing by $t$ and taking liminf we obtain

$$
\liminf _{t \rightarrow 0} \frac{E_{x_{0}}^{v}(\sigma)-E_{x_{0}}^{v_{t \eta}}(\sigma)}{t} \leq C \int_{B_{\sigma}\left(x_{0}\right)} \eta d^{2}\left(v(x), P_{0}\right) d \mu .
$$

Combining this with (45) and letting $\eta$ approximate the characteristic function of $B_{\sigma}\left(x_{0}\right)$, we obtain

$$
2 E_{x_{0}}^{v}(\sigma) \leq \int_{\partial B_{\sigma}\left(x_{0}\right)} \frac{\partial}{\partial r} d^{2}\left(v(x), P_{0}\right) d \Sigma+C \int_{B_{\sigma}\left(x_{0}\right)} d^{2}\left(v(x), P_{0}\right) d \mu .
$$

Now Lemma 23 completes the proof. Q.E.D.

\section{Order Function}

We continue with the analysis of the singular component map and start with the following Proposition on the lower bound of the order function.

Proposition 25 Let $u=(V, v): B_{\sigma_{\star}}\left(x_{\star}\right) \rightarrow\left(\mathbf{R}^{j} \times Y_{2}{ }^{k-j}, G\right)$ satisfy Assumption 20. Then for any $\epsilon_{0}>0$, there exists $R_{0}>0$ such that

$$
1-\epsilon_{0} \leq \frac{\sigma E_{x_{0}}^{v}(\sigma)}{I_{x_{0}}^{v}(\sigma)}, \forall x_{0} \in \mathcal{S}_{j}(u) \cap B_{\frac{\sigma_{\star}}{2}}\left(x_{\star}\right) \text { and } \forall \sigma \in\left(0, R_{0}\right] .
$$

Proof. If (47) is not true, then there exist a sequence $x_{i} \in \mathcal{S}_{j}(u)$ and $r_{i} \rightarrow 0$ such that

$$
\frac{r_{i} E_{x_{i}}^{v}\left(r_{i}\right)}{I_{x_{i}}^{v}\left(r_{i}\right)}<1-\epsilon_{0}
$$


Note here that $x_{i} \in \mathcal{S}_{j}(u)$ implies $v\left(x_{i}\right)=P_{0}$ for all $i$. For each $x_{i}$, use normal coordinates to identify a $r_{i}$-ball about $x_{i}$ with $\left(B_{r_{i}}(0), g_{x_{i}}\right)$ where $B_{r_{i}}(0) \subset \mathbf{R}^{n}$. We define the restriction maps

$$
{ }_{i} v:\left(B_{r_{i}}(0), g_{x_{i}}\right) \rightarrow Y_{2}^{k-j}, \quad{ }_{i} v=\left.v\right|_{B_{r_{i}}(0)}
$$

and the harmonic maps

$$
{ }_{i} w:\left(B_{r_{i}}(0), g_{x_{i}}\right) \rightarrow Y_{2}^{k-j} \text { with }\left.{ }_{i} w\right|_{\partial B_{r_{i}}(0)}=\left.{ }_{i} v\right|_{\partial B_{r_{i}}(0)}
$$

with respect to the metric $h$. Let

$$
\nu_{i}=\left(\frac{I_{0}^{i v}\left(r_{i}\right)}{r_{i}^{n-1}}\right)^{1 / 2}
$$

Let $g_{i}(x)=g_{x_{i}}\left(r_{i} x\right)$ be the metric given on $B_{1}(0)$ and define the rescaled maps

$$
v_{i}, w_{i}:\left(B_{1}(0), g_{i}\right) \rightarrow\left(Y_{2}^{k-j}, h\right)
$$

by setting

$$
v_{i}(x)=\nu_{i}^{-1}{ }_{i} v\left(r_{i} x\right) \text { and } w_{i}(x)=\nu_{i}^{-1}{ }_{i} w\left(r_{i} x\right)
$$

The normalization by $\nu_{i}$ implies that

$$
I_{0}^{v_{i}}(1)=I_{0}^{w_{i}}(1)=1 .
$$

In particular, (48) guarantees that

$$
E_{0}^{w_{i}}(1) \leq E_{0}^{v_{i}}(1)=\frac{E_{0}^{v_{i}}(1)}{I_{0}^{v_{i}}(1)} \leq 1-\epsilon_{0}
$$

Therefore, $\left\{w_{i}\right\}$ is a sequence of harmonic maps with $I_{0}^{w_{i}}(1)=1$ and uniformly bounded energy. For any $r \in(0,1)$, the Lipschitz constant for $\left\{w_{i}\right\}$ in $B_{r}(0)$ depends on the energy bound and $r$ and is independent of $i$ (cf. Theorem 2.4.6 [KS1]). By Arzela-Ascoli, there exists a subsequence of $\left\{w_{i}\right\}$ (which we still denote by $\left\{w_{i}\right\}$ by an abuse of notation) converging uniformly in the pull-back sense on every compact set to a map

$$
v_{0}:\left(B_{1}(0), \delta\right) \rightarrow\left(Y_{2}^{k-j}, h\right)
$$


where $\delta$ is the standard Euclidean metric on $B_{1}(0)$ and

$$
E_{0}^{v_{0}}(\sigma)=\lim _{i \rightarrow \infty} E_{0}^{w_{i}}(\sigma), \forall \sigma \in(0,1)
$$

The map $v_{0}$ is non-constant since $I_{0}^{w_{i}}(1)=1$ by the $L^{2}$-trace theorem (cf. Theorem 1.12 .2 of $[\mathrm{KS} 1]$ ). The fact that $v_{0}$ is energy minimizing on every compact subset of $B_{1}(0)$ immediately implies $v_{0}$ is energy minimizing on $B_{1}(0)$ by the same argument as in Lemma 11 . Since

$$
\int_{\partial B_{1}(0)} d^{2}\left(v_{i}, P_{0}\right) d \mu=1 \text { and } E_{0}^{v_{i}}(1) \leq\left(1-\epsilon_{0}\right),
$$

Theorems 1.13 and 1.12.2 of [KS1] imply that the sequences $\left\{v_{i}\right\}$ (resp. $\left.\left\{\left.v_{i}\right|_{\partial B_{1}(0)}\right\}\right)$ converge in $L^{2}$ to a map which we claim to be $v_{0}$ (resp. $\left.\left.v_{0}\right|_{\partial B_{1}(0)}\right)$. Indeed we will now show that

$$
\int_{B_{1}(0)} d^{2}\left(v_{i}, v_{0}\right) d \mu \rightarrow 0
$$

To see this, let ${ }_{i} v_{\frac{1}{2}}=\frac{1}{2}{ }_{i} v+\frac{1}{2}{ }_{i} w$. Then

$$
\begin{aligned}
& \int_{B_{r_{i}}(0)}\left|\nabla d\left({ }_{i} v,,_{i} w\right)\right|^{2} d \mu \\
& \quad \leq E_{0}^{i v}\left(r_{i}\right)+E_{0}^{i w}\left(r_{i}\right)-2 E_{0}^{i v \frac{1}{2}}\left(r_{i}\right) \quad(\text { by }[\mathrm{KS} 1](2.2 i v)) \\
& \quad \leq E_{0}^{i v}\left(r_{i}\right)-E_{0}^{i w}\left(r_{i}\right) .
\end{aligned}
$$

Thus, Proposition 22, Lemma 23 and (48) gives us

$$
\int_{B_{r_{i}}(0)}\left|\nabla d\left({ }_{i} v,{ }_{i} w\right)\right|^{2} d \mu \leq C r_{i} I_{0}^{i v}\left(r_{i}\right)
$$

Using Poincare inequality $\int_{B_{r_{i}}(0)} d^{2}\left({ }_{i} v,_{i} w\right) d \mu \leq C r_{i}^{2} \int_{B_{r_{i}}(0)}\left|\nabla d\left({ }_{i} v,_{i} w\right)\right|^{2} d \mu$, we obtain

$$
\int_{B_{r_{i}}(0)} d^{2}\left({ }_{i} v,{ }_{i} w\right) d \mu \leq C r_{i}^{3} I_{0}^{i v}\left(r_{i}\right)
$$

Using a change of variables $\phi_{i}: B_{1}(0) \rightarrow B_{r_{i}}(0)$ defined by $\phi_{i}(x)=r_{i} x$, we obtain

$$
\int_{B_{1}(0)} d^{2}\left({ }_{i} v \circ \phi_{i},{ }_{i} w \circ \phi_{i}\right) d \mu \leq C r_{i}^{2} I_{0}^{i v \circ \phi_{i}}(1) .
$$


The loss of $r_{i}$ on the first term on the right-hand side of (52) after the change of variables comes from the fact that $I^{i v}$ is an expression involving $(n-1)$-dimensional integral compared to the left-hand side of (52) which is an expression involving a $n$-dimensional integral. Now multiply both sides by $\nu_{i}^{-2}$ to obtain

$$
\int_{B_{1}(0)} d^{2}\left(v_{i}, w_{i}\right) d \mu \leq C r_{i}^{2} I_{0}^{v_{i}}(1)=C r_{i}^{2}
$$

Therefore as $i \rightarrow \infty$,

$$
\int_{B_{1}(0)} d^{2}\left(v_{i}, v_{0}\right) d \mu \leq 2 \int_{B_{1}(0)} d^{2}\left(v_{i}, w_{i}\right) d \mu+2 \int_{B_{1}(0)} d^{2}\left(w_{i}, v_{0}\right) d \mu \rightarrow 0
$$

and this proves (51).

Using the facts that the order of a harmonic map is $\geq 1$, the $L^{2}$-trace theorem (cf. Theorem 1.12.2 [KS1]) which implies $I_{0}^{v_{0}}(1)=\lim _{i \rightarrow \infty} I_{0}^{v_{i}}(1)$ and the lower semicontinuity of the energy $E_{0}^{v_{0}}(1) \leq \liminf _{i \rightarrow \infty} E_{0}^{v_{i}}(1)$, we obtain

$$
1 \leq \frac{E_{0}^{v_{0}}(1)}{I_{0}^{v_{0}}(1)} \leq \liminf _{i \rightarrow 0} \frac{E_{0}^{v_{i}}(1)}{I_{0}^{v_{i}}(1)} \leq 1-\epsilon_{0}
$$

This is a contradiction. Q.E.D.

The next Lemma is the analogue of the domain variation formula (2.3) of $[\mathrm{GS}]$

Lemma 26 Let $u=(V, v): B_{\sigma_{\star}}\left(x_{\star}\right) \rightarrow\left(\mathbf{R}^{j} \times Y_{2}^{k-j}, G\right)$ satisfy Assumption 20. There exists $R_{0}>0$ such that for $x_{0} \in \mathcal{S}_{j}(u) \cap B \frac{\sigma_{\star}}{2}\left(x_{\star}\right)$ and $\sigma \in\left(0, R_{0}\right)$, we have

$$
\left(2-n+O\left(\sigma^{2}\right)\right) E_{x_{0}}^{v}(\sigma)+\sigma \int_{\partial B_{\sigma}\left(x_{0}\right)}|\nabla v|^{2} d \Sigma-2 \sigma \int_{\partial B_{\sigma}\left(x_{0}\right)}\left|\frac{\partial v}{\partial r}\right|^{2} d \mu \geq 0 .
$$

Proof. By (46) and Lemma 23, we obtain

$$
\begin{aligned}
\liminf _{t \rightarrow 0} \frac{E_{x_{0}}^{v}(\sigma)-E_{x_{0}}^{v t \eta}(\sigma)}{t} & \leq C \int_{B_{\sigma}\left(x_{0}\right)} \eta d^{2}\left(v, P_{0}\right) d \mu \\
& \leq C\left(\sigma I_{x_{0}}^{v}(\sigma)+\sigma^{2} E_{x_{0}}^{v}(\sigma)\right) .
\end{aligned}
$$


Combining with Proposition 25, for $\sigma<R_{0}$ we have

$$
\liminf _{t \rightarrow 0} \frac{E_{x_{0}}^{v}(\sigma)-E_{x_{0}}^{v_{t \eta}}(\sigma)}{t} \leq C \sigma^{2} E_{x_{0}}^{v}(\sigma)
$$

Now as in [GS] p.192-193, we also have

$$
\begin{aligned}
& \lim _{t \rightarrow 0} \frac{E_{x_{0}}^{v_{t \eta}}(\sigma)-E_{x_{0}}^{v}(\sigma)}{t} \\
& \quad=\left(2-n+O\left(\sigma^{2}\right)\right) E_{x_{0}}^{v}(\sigma)+\sigma \int_{\partial B_{\sigma}\left(x_{0}\right)}|\nabla v|^{2} d \Sigma-2 \sigma \int_{\partial B_{\sigma}\left(x_{0}\right)}\left|\frac{\partial v}{\partial r}\right|^{2} d \mu
\end{aligned}
$$

which combined with the previous inequality implies the Lemma. Q.E.D.

The next result is to prove the following existence property of the order for the singular component of a harmonic map.

Proposition 27 Let $u=(V, v): B_{\sigma_{\star}}\left(x_{\star}\right) \rightarrow\left(\mathbf{R}^{j} \times Y_{2}{ }^{k-j}, G\right)$ satisfy Assumption 20. For $x_{0} \in \mathcal{S}_{j}(u) \cap B \frac{\sigma_{\star}}{2}\left(x_{\star}\right)$ and $0<\sigma<\sigma_{0}=: \sup \left\{\sigma: B_{\sigma}\left(x_{0}\right) \subset\right.$ $\left.B_{\sigma_{\star}}\left(x_{\star}\right)\right\}$, define

$$
\operatorname{Ord}^{v}\left(x_{0}, \sigma\right):=\frac{\sigma E_{x_{0}}^{v}(\sigma)}{I_{x_{0}}^{v}(\sigma)} .
$$

Then

$$
\operatorname{Ord}^{v}\left(x_{0}\right):=\lim _{\sigma \rightarrow 0} \operatorname{Ord}^{v}\left(x_{0}, \sigma\right) \text { exists. }
$$

In fact, there exist constants $C>0, C_{1}>0$ and $R_{0}>0$ such that for any $x_{0} \in \mathcal{S}_{j}(u) \cap B_{\frac{\sigma_{\star}}{2}}\left(x_{\star}\right)$, there is a function $\sigma \mapsto F_{x_{0}}(\sigma)$ with the properties that

$$
I_{x_{0}}^{v}(\sigma) \leq F_{x_{0}}(\sigma) \leq I_{x_{0}}^{v}(\sigma) e^{C_{1} \sigma^{2}}
$$

and

$$
\sigma \mapsto e^{C \sigma^{2}} \frac{\sigma E_{x_{0}}^{v}(\sigma)}{F_{x_{0}}(\sigma)} \text { is non-decreasing in }\left(0, R_{0}\right) \text {. }
$$

Proof. By Proposition 25, there exists $R_{0}>0$ such that

$$
\frac{1}{2} \leq \frac{\sigma E_{x_{0}}^{v}(\sigma)}{I_{x_{0}}^{v}(\sigma)}, \forall \sigma \in\left(0, R_{0}\right)
$$


for any $x_{0} \in \mathcal{S}_{j}\left(x_{0}\right)$. Now fix $x_{0} \in \mathcal{S}_{j}\left(x_{0}\right)$. For notational simplicity, let $I(\sigma)=I_{x_{0}}^{v}(\sigma)$ and $E(\sigma)=E_{x_{0}}^{v}(\sigma)$. Proposition 24 and (55) then imply that

$$
2 E(\sigma) \leq \int_{\partial B_{\sigma}\left(x_{0}\right)} \frac{\partial}{\partial r} d^{2}\left(v, P_{0}\right) d \mu+C \sigma^{2} E(\sigma), \sigma \in\left(0, R_{0}\right)
$$

for some constant $C$ independent of $x_{0}$. By Lemma 26 and the identity

$$
I^{\prime}(\sigma)=\int_{\partial B_{\sigma}\left(x_{0}\right)} \frac{\partial}{\partial r} d^{2}\left(v, P_{0}\right) d \Sigma+\frac{n-1}{\sigma} I(\sigma)+O(\sigma) I(\sigma)
$$

(cf. [GS] p.193), we obtain

$$
\begin{aligned}
& \frac{I^{\prime}(\sigma)}{I(\sigma)}-\frac{E^{\prime}(\sigma)}{E(\sigma)}-\frac{1}{\sigma} \\
& \quad=\frac{\left(E(\sigma) \int_{\partial B_{\sigma}\left(x_{0}\right)} \frac{\partial}{\partial r} d^{2}\left(v, P_{0}\right) d \Sigma-2 I(\sigma) \int_{\partial B_{\sigma}\left(x_{0}\right)}\left|\frac{\partial v}{\partial r}\right|^{2} d \Sigma\right)}{E(\sigma) I(\sigma)}+O(\sigma) .
\end{aligned}
$$

The derivation of (58) is exactly as in [GS]. We note that the $O(\sigma)$ term is only dependent on the domain metric and can be bounded by a constant independent of $x_{0} \in \Omega$. Combining (56) and (57), we obtain

$$
\begin{aligned}
E(\sigma) & \int_{\partial B_{\sigma}\left(x_{0}\right)} \frac{\partial}{\partial r} d^{2}\left(v, P_{0}\right) d \Sigma-2 I(\sigma) \int_{\partial B_{\sigma}\left(x_{0}\right)}\left|\frac{\partial v}{\partial r}\right|^{2} d \Sigma \\
\leq & \frac{1}{2}\left(\int_{\partial B_{\sigma}\left(x_{0}\right)} \frac{\partial}{\partial r} d^{2}\left(v, P_{0}\right) d \Sigma\right)^{2}+C \sigma^{2} E(\sigma)\left(\int_{\partial B_{\sigma}\left(x_{0}\right)} \frac{\partial}{\partial r} d^{2}\left(v, P_{0}\right) d \Sigma\right) \\
& -2 I(\sigma) \int_{\partial B_{\sigma}\left(x_{0}\right)}\left|\frac{\partial v}{\partial r}\right|^{2} d \Sigma \\
\leq & \frac{1}{2}\left(\int_{\partial B_{\sigma}\left(x_{0}\right)} \frac{\partial}{\partial r} d^{2}\left(v, P_{0}\right) d \Sigma\right)^{2}-2 I(\sigma) \int_{\partial B_{\sigma}\left(x_{0}\right)}\left|\frac{\partial v}{\partial r}\right|^{2} d \Sigma \\
& +C \sigma^{2} E(\sigma)\left(I^{\prime}(\sigma)-\frac{n-1}{\sigma} I(\sigma)+C \sigma I(\sigma)\right) .
\end{aligned}
$$

Since

$$
\left|\frac{\partial}{\partial r} d\left(v, P_{0}\right)\right|^{2} \leq\left|\frac{\partial v}{\partial r}\right|^{2}
$$


we have

$$
\begin{aligned}
& \frac{1}{2}\left(\int_{\partial B_{\sigma}\left(x_{0}\right)} \frac{\partial}{\partial r} d^{2}\left(v, P_{0}\right) d \Sigma\right)^{2}-2 I(\sigma) \int_{\partial B_{\sigma}\left(x_{0}\right)}\left|\frac{\partial v}{\partial r}\right|^{2} d \Sigma \\
& \quad=2\left(\int_{\partial B_{\sigma}\left(x_{0}\right)} d\left(v, P_{0}\right) \frac{\partial}{\partial r} d\left(v, P_{0}\right) d \Sigma\right)^{2}-2 I(\sigma) \int_{\partial B_{\sigma}\left(x_{0}\right)}\left|\frac{\partial v}{\partial r}\right|^{2} d \Sigma \\
& \quad \leq 2 I(\sigma)\left(\int_{\partial B_{\sigma}\left(x_{0}\right)}\left|\frac{\partial}{\partial r} d\left(v, P_{0}\right)\right|^{2} d \Sigma\right)-2 I(\sigma) \int_{\partial B_{\sigma}\left(x_{0}\right)}\left|\frac{\partial v}{\partial r}\right|^{2} d \Sigma \\
& \quad \leq 0 .
\end{aligned}
$$

Thus, by combining (58) with (59) and (60), we obtain

$$
0 \leq \frac{E^{\prime}(\sigma)}{E(\sigma)}+\frac{1}{\sigma}-\left(1-C \sigma^{2}\right) \frac{I^{\prime}(\sigma)}{I(\sigma)}+C \sigma, \quad \text { for a.e. } \sigma \in\left(0, \rho_{0}\right)
$$

for $C>0$ sufficiently large. Here we note that $C$ depends only on the domain and the target metric and hence can be chosen independently of $x_{0}$. Inequality (61) is (15) in [Me]. If we set

$$
F_{x_{0}}(\sigma)=I(\sigma) \exp \left(C \int_{\sigma}^{\sigma_{0}} s^{2} \frac{d}{d s} \log I(s) d s\right),
$$

then we can deduce that $F_{x_{0}}(\sigma)$ satisfies $(53)$ and (54) from the proof of Proposition 3.1 of $[\mathrm{Me}]$. Furthermore, $C_{1}>0$ can be chosen independently of $x_{0}$. Q.E.D.

We can now define blow up maps and a tangent map of the singular component map $v$ of $u$. Let $u=(V, v): B_{\sigma_{\star}}\left(x_{\star}\right) \rightarrow\left(\mathbf{R}^{j} \times Y_{2}{ }^{k-j}, G\right)$ satisfy Assumption 20. Fix $x_{0} \in \mathcal{S}_{j}(u) \cap B_{\frac{\sigma_{\star}}{2}}\left(x_{\star}\right)$ and use normal coordinates at $x_{0}=0$. Let

$$
v_{i}:\left(B_{1}(0), g_{i}\right) \rightarrow\left(Y_{2}^{k-j}, h\right)
$$

be defined as in Proposition 25 with $x_{i}=x_{0}$ for all $i$. By construction, we have $I_{0}^{v_{i}}(1)=1$. By Proposition 27,

$$
E_{0}^{v_{i}}(1)=\frac{E_{0}^{v_{i}}(1)}{I_{0}^{v_{i}}(1)}=\frac{r_{i} E_{0}^{v}\left(r_{i}\right)}{I_{0}^{v}\left(r_{i}\right)} \leq \operatorname{Ord}^{v}(0)+1
$$


for $i$ sufficiently large. Replacing inequality (50) by the above inequality, we can apply the argument in the proof of Proposition 25 to assert the existence of a sequence of $\left\{v_{i}\right\}$ (resp $\left.\left\{\left.v_{i}\right|_{\partial B_{1}(0)}\right\}\right)$ converging in $L^{2}$ to a map $v_{0}: B_{1}(0) \rightarrow\left(Y_{2}^{k-j}, h\right)$ (resp. $\left.\left.v_{0}\right|_{\partial B_{1}(0)}\right)$. Therefore, we have

$$
E_{0}^{v_{0}}(r) \leq \liminf _{i \rightarrow \infty} E_{0}^{v_{i}}(r), r \in(0,1]
$$

by the lower semicontinuity of energy (cf. Theorem 1.6 [KS1]) and

$$
I_{0}^{v_{0}}(1)=\lim _{i \rightarrow \infty} I_{0}^{v_{i}}(1), r \in(0,1]
$$

by the $L^{2}$ trace theorem (cf. Theorem 1.12.2 [KS1]). Furthermore, $v_{0}$ is a harmonic map (since it is the limit of harmonic maps $\left\{w_{i}\right\}$ ).

Definition 28 The maps $v_{i}$ and $v_{0}$ above are called blow-up maps and a tangent map of $v$ at $x_{0}$.

The following results are corollaries of Proposition 27.

Corollary 29 Let $u=(V, v): B_{\sigma_{\star}}\left(x_{\star}\right) \rightarrow\left(\mathbf{R}^{j} \times Y_{2}^{k-j}, G\right)$ satisfy Assumption 20. If $v \equiv P_{0}$ on any open subset of $B_{\frac{\sigma_{\star}}{2}}\left(x_{\star}\right)$, then $v \equiv P_{0}$ in $B_{\frac{\sigma_{\star}}{2}}\left(x_{\star}\right)$.

Proof. If $v$ is not constant in $B_{\frac{\sigma_{\star}}{2}}\left(x_{\star}\right)$ but identically equal to $P_{0}$ on an open subset of $B \frac{\sigma_{\star}}{2}\left(x_{\star}\right)$, then there exists a ball $B \subset B \frac{\sigma_{\star}}{2}\left(x_{\star}\right)$ such that $v \equiv P_{0}$ in the interior of $B$, but for some $x_{0} \in \partial B, v$ is not constant in any neighborhood of $x_{0}$. Let $v_{0}: B_{1}(0) \rightarrow\left(Y_{2}^{k-j}, h\right)$ be the tangent map of $v$ at $x_{0}$. Then $v_{0}$ is identically constant on half of $B_{1}(0)$ and this contradicts Proposition 3.4 [GS]. Q.E.D.

Corollary 30 Let $u=(V, v): B_{\sigma_{\star}}\left(x_{\star}\right) \rightarrow\left(\mathbf{R}^{j} \times Y_{2}{ }^{k-j}, G\right)$ satisfy Assumption 20. Then, there exists $A>0$ such that for $x_{0} \in \mathcal{S}_{j}(u) \cap B_{\frac{\sigma_{\star}}{2}}\left(x_{\star}\right)$, we have

$$
\operatorname{Ord}^{v}\left(x_{0}\right) \leq A
$$

Proof. Since

$$
\int_{\sigma}^{\sigma_{0}} s^{2} \frac{d}{d s} \log I(s) d s=\sigma_{0}^{2} \log I\left(\sigma_{0}\right)-\sigma^{2} \log I(\sigma)-2 \int_{\sigma}^{\sigma_{0}} s \log I(s) d s,
$$


the map $x_{0} \mapsto F_{x_{0}}(\sigma)$ is a continuous map. Since $F_{x_{0}}(\sigma) \neq 0$ by Corollary 29, the map $x_{0} \mapsto \frac{\sigma E_{x_{0}}^{v}(\sigma)}{F_{x_{0}}(\sigma)}$ is also continuous. Thus, the result follows from the fact that a non-increasing limit of continuous functions is upper semicontinuous. Q.E.D.

Corollary 31 Let $u=(V, v): B_{\sigma_{\star}}\left(x_{\star}\right) \rightarrow\left(\mathbf{R}^{j} \times Y_{2}{ }^{k-j}, G\right)$ satisfy Assumption 20. Then, there exist $C>0$ and $R_{0}>0$ such that for any $x_{0} \in \mathcal{S}_{j}(u) \cap B \frac{\sigma_{\star}}{2}\left(x_{\star}\right)$, we have

$$
\sigma \mapsto e^{C \sigma^{2}} \frac{I_{x_{0}}^{v}(\sigma)}{\sigma^{n-1+2 \alpha}} \text { and } \sigma \mapsto e^{C \sigma^{2}} \frac{E_{x_{0}}^{v}(\sigma)}{\sigma^{n-2+2 \alpha}}
$$

is monotone non-decreasing in $\left(0, R_{0}\right)$, where $\alpha=\operatorname{Ord}^{v}\left(x_{0}\right)$.

Proof. Let $I(\sigma)=I_{x_{0}}^{v}(\sigma), E(\sigma)=E_{x_{0}}^{v}(\sigma)$ and $F(\sigma)=F_{x_{0}}(\sigma)$. By Propositions 24 and 27,

$$
\begin{gathered}
e^{-C \sigma^{2}} \alpha I(\sigma) \leq e^{-C \sigma^{2}} \alpha F(\sigma) \leq \sigma E(\sigma) \\
\leq \frac{\sigma}{2}\left(\int_{\partial B_{\sigma}\left(x_{0}\right)} \frac{\partial}{\partial r} d^{2}\left(v, P_{0}\right)+C \sigma I(\sigma)\right) \\
\leq \frac{1}{2-C \sigma^{2}}\left(\sigma I^{\prime}(\sigma)-(n-1) I(\sigma)+C \sigma^{2} I(\sigma)\right),
\end{gathered}
$$

for some $C>0$ (which depends on the constants in Propositions 24 and 27 and can be chosen independently of $\left.x_{0} \in B_{\frac{\sigma_{\star}}{2}}\left(x_{\star}\right)\right)$. Thus, by adjusting $\mathrm{C}$ and absorbing all the error in the $C \sigma$ term we have

$$
\frac{I^{\prime}(\sigma)}{I(\sigma)} \geq \frac{n-1+2 \alpha}{\sigma}-C \sigma
$$

for $\sigma>0$ sufficiently small. Similarly, using inequality (61), we have

$$
\frac{E^{\prime}(\sigma)}{E(\sigma)} \geq \frac{n-2+2 \alpha}{\sigma}-C \sigma
$$

and this immediately implies the assertion of the Corollary. Q.E.D. 


\section{The Gap Property of the Order}

We start by stating the $\epsilon$-regularity theorem of [DM].

Theorem 32 Let $Y$ be locally compact Riemannian simplicial complex and assume $Y_{0}$ is a totally geodesic subcomplex of $Y$. Fix $P \in Y_{0}$ and assume that $T_{P_{1}} Y_{0}$ is essentially regular for $P_{1} \in Y_{0}$ close to $P$. Let

$$
l_{0}: B_{r_{0}}(0) \subset \mathbf{R}^{n} \rightarrow T_{P} Y
$$

be an instrinsically homogeneous degree 1 map effectively contained in $T_{P} Y_{0}$ and $l_{0}(0)=\mathcal{O}_{P}$, the origin of $T_{P} Y$. Let $\Omega$ be a smooth Riemannian domain, $u: \Omega \rightarrow Y$ a harmonic map, $\Omega_{0}$ a compactly contained subset of $\Omega$ and $x_{0} \in \Omega_{0}$ such that $u\left(x_{0}\right)=P$ and $u$ is of order 1 at $x_{0}$. There exist $\sigma_{0}>0$ and $\delta_{0}>0$ such that if

$$
\frac{1}{\sigma^{n}} \int_{B_{\sigma}(0)} d\left(\exp _{P}^{-1} \circ u \circ \exp _{x_{0}}(x), l_{0}(x)\right) d \mu<\delta_{0}, \forall \sigma \in\left(0, \sigma_{0}\right)
$$

then $u\left(B_{\sigma^{\prime}}\left(x_{0}\right)\right) \subset Y_{0}$ for some $\sigma^{\prime}>0$. The constants $\sigma_{0}$ and $\delta_{0}$ depend only on $\Omega_{0}, \Omega, Y_{0}$ and the total energy $E^{u}$ of $u$.

For the definitions of effectively contained and essentially regular, we refer to $[\mathrm{GS}]$ or $[\mathrm{DM}]$. This immediately implies the following

Corollary 33 Let $\Omega$ be an n-dimensional smooth Riemannian domain, $Y$ an $m$-dimensional locally compact Riemannian simplicial complex, $u: \Omega \rightarrow Y a$ harmonic map and $x_{0} \in \Omega$ with $\operatorname{Ord}\left(x_{0}\right)=1$. There exists a totally geodesic subcomplex $Y_{0}$ which is isometric to the twisted product $\left(\mathbf{R}^{m} \times Y_{2}^{k-m}, G\right)$ for some integer $m$ with $1 \leq m \leq \min \{n, k\}$ and some $D M$ complex $Y_{2}^{k-m}$ of dimension $k-m$ such that $u\left(B_{\sigma^{\prime}}\left(x_{0}\right)\right) \subset Y_{0}$ for some $\sigma^{\prime}>0$.

Proof. By Lemma 6.2 of [GS] the tangent map $u_{*}$ at $x_{0}$ is effectively contained into a subcomplex $\mathbf{R}^{m} \times X_{0}^{k-m} \subset T_{P} Y, P=u\left(x_{0}\right)$. Apply Theorem 32 to $Y_{0}=\exp _{P}\left(\mathbf{R}^{m} \times X_{0}^{k-m}\right)$ and $l_{0}=u_{*}$. Q.E.D.

Recall that by the $\epsilon$-gap Theorem of [GS], given any harmonic map $w$ : $B_{1}(0) \subset \mathbf{R}^{n} \rightarrow\left(Y_{2}, h\right)$ with $w(0)=P_{0}$, either

$$
\operatorname{Ord}^{w}(0)=1 \text { or } \operatorname{Ord}^{w}(0) \geq 1+\epsilon_{0}
$$

where $\epsilon_{0}>0$ depends only on $h$. We will now prove that this gap property holds for the singular component map of a harmonic map. 
Proposition 34 Let $u=(V, v): B_{\sigma_{\star}}\left(x_{\star}\right) \rightarrow\left(\mathbf{R}^{j} \times Y_{2}{ }^{k-j}, G\right)$ satisfy Assumption 20. There exists $\epsilon_{0}>0$ such that

$$
\operatorname{Ord}^{v}\left(x_{0}\right) \in\left[1+\epsilon_{0}, \infty\right), \forall x_{0} \in \mathcal{S}_{j}(u) .
$$

Proof. Let $\left\{v_{i}\right\}$ and $v_{0}$ be blow up maps and a tangent map of $v$ at $x_{0}=0$ of Definition 28. Thus, we obtain for any $\sigma \in(0,1]$,

$$
\begin{aligned}
\operatorname{Ord}^{v_{0}}(0) & \left.\leq \frac{\sigma E_{0}^{v_{0}}(\sigma)}{I_{0}^{v_{0}}(\sigma)} \text { (Monotonicity Formula, [GS] equation }(2.5)\right) \\
& \leq \liminf _{i \rightarrow \infty} \frac{\sigma E_{0}^{v_{i}}(\sigma)}{I_{0}^{v_{i}}(\sigma)}(\text { by }(62) \text { and }(63)) \\
& =\liminf _{i \rightarrow \infty} \frac{r_{i} \sigma E_{0}^{v}\left(r_{i} \sigma\right)}{I_{0}^{v}\left(r_{i} \sigma\right)} \\
& =\operatorname{Ord}^{v}(0)
\end{aligned}
$$

If $\operatorname{Ord}^{v_{0}}(0)=1$ the tangent map $v_{0 *}$ of $v_{0}$ at 0 is a degree 1 , homogeneous map. Hence $v_{0 *}$ maps into a flat $F$ of $Y_{2}^{k-j}$ (cf. Proposition 3.1 [GS]). Let $Y_{2}^{\prime}$ be the union of all $(k-j)$-flats containing $F$. Then map $v_{0 *}$ is homogeneous of degree 1 and effectively contained in $Y_{2}^{\prime}$ and we can write $Y_{2}^{\prime}=\mathbf{R}^{l} \times Y_{3}^{k-j-l}$ where $l$ is the dimension of $F$ and $Y_{3}^{k-j-l}$ is a F-connected complex of dimension $k-j-l$ (cf. Lemma 6.2 [GS]). If we let $U_{*}=\left(u_{*}, v_{0 *}\right)$ where $u_{*}$ is the tangent map of $u$ at $x_{0}$, then $U_{*}$ a homogeneous, degree 1 map is effectively contained in $\mathbf{R}^{j} \times \mathbf{R}^{l} \times Y_{3}^{k-j-l}$. Note that $u_{*}$ maps into the first component of $\mathbf{R}^{j} \times Y_{2}^{k-j}$. Therefore, given any $\delta_{0}>0$, we can choose $i$ sufficiently large such that

$$
\frac{1}{r_{i}^{n}} \int_{B_{r_{i}}(0)} d\left(V, u_{*}\right) d \mu<\frac{\delta_{0}}{2}
$$

and

$$
\begin{aligned}
\frac{1}{r_{i}^{n}} \int_{B_{r_{i}}(0)} d\left(v, v_{0 *}\right) d \mu & \leq \frac{1}{r_{i}^{n}} \int_{B_{r_{i}}(0)}\left(d\left(v, v_{0}\right)+d\left(v_{0}, v_{0 *}\right)\right) d \mu \\
& \leq \nu_{i} \int_{B_{1}(0)} d_{i}\left(v_{i}, v_{0}\right) d \mu+\frac{1}{r_{i}^{n}} \int_{B_{r_{i}}(0)} d\left(v_{0}, v_{0 *}\right) d \mu \\
& \leq \nu_{i} \int_{B_{1}(0)} d_{i}\left(v_{i}, v_{0}\right) d \mu+\frac{1}{r_{i}^{n}} \int_{B_{r_{i}}(0)} d\left(v_{0}, v_{0 *}\right) d \mu \\
& <\frac{\delta_{0}}{2}
\end{aligned}
$$


Thus,

$\frac{1}{r_{i}^{n}} \int_{B_{r_{i}}\left(x_{0}\right)} d\left(u, U_{*}\right) d \mu \leq \frac{1}{r_{i}^{n}} \int_{B_{r_{i}}\left(x_{0}\right)} d\left(V, u_{*}\right) d \mu+\frac{1}{r_{i}^{n}} \int_{B_{r_{i}}\left(x_{0}\right)} d\left(v, v_{0 *}\right) d \mu<\delta_{0}$.

By Corollary 33, there exists $\sigma>0$ such that

$$
u\left(B_{\sigma}\left(x_{0}\right)\right) \subset \mathbf{R}^{j+l} \times Y_{3}^{k-j-l}
$$

and this contradicts that $x_{0} \in \mathcal{S}_{j}(u)$. Thus, $\operatorname{Ord}^{v_{0}}(0)>1$. By (64), there exists $\epsilon_{0}>0$ such that $1+\epsilon_{0} \leq \operatorname{Ord}^{v_{0}}(0) \leq \operatorname{Ord}^{v}\left(x_{0}\right)$. Q.E.D.

\section{Proof of the Main Theorem}

By induction it suffices to show that if $u=(V, v): B_{\sigma_{\star}}\left(x_{\star}\right) \rightarrow\left(\mathbf{R}^{j} \times Y_{2}^{k-j}, G\right)$ is a harmonic map with its image contained in a good neighborhood of $u\left(x_{\star}\right)=\left(0, P_{0}\right), G\left(u\left(x_{\star}\right)\right)=H \times h$ on $\mathbf{R}^{j} \times Y_{2}^{k-j}$ defines a $F$-connected complex and $\operatorname{dim}_{\mathcal{H}} \mathcal{S}_{j+1}^{+}(u) \leq n-2$, then $\operatorname{dim}_{\mathcal{H}}\left(\mathcal{S}_{j}(u)\right) \leq n-2$.

Following [GS] we define the rough outer Hausdorff measure $\hat{\mathcal{H}}^{s}(\cdot)$ by

$$
\hat{\mathcal{H}}^{s}(S)=\inf \left\{\sum_{l=1}^{\infty} r_{l}^{s}: \text { all coverings }\left\{B_{r_{l}}\left(x_{l}\right)\right\}_{l=1}^{\infty} \text { of } S \text { by open balls }\right\} .
$$

$\hat{\mathcal{H}}^{s}$ is clearly an outer measure which is not precisely the Hausdorff measure $\mathcal{H}^{s}$. On the other hand, for any set $S$ the Hausdorff dimension is given by

$$
\operatorname{dim}_{\mathcal{H}}(S)=\inf \left\{s: \mathcal{H}^{s}(S)=0\right\}=\inf \left\{s: \hat{\mathcal{H}}^{s}(S)=0\right\} .
$$

Now assume by contradiction that $\operatorname{dim}_{\mathcal{H}}\left(\mathcal{S}_{j}(u)\right)>n-2$. By $[\mathrm{Fe}] 2.10 .19$, there exists $s_{0}>n-2, x_{0} \in \mathcal{S}_{j}(u)$ and a sequence $\sigma_{i} \rightarrow 0$ such that

$$
\begin{aligned}
\lim _{i \rightarrow \infty} \sigma_{i}^{-s_{0}} \hat{\mathcal{H}}^{s_{0}}\left(\mathcal{S}_{j}(u) \cap \overline{B_{\frac{\sigma_{i}}{2}}\left(x_{0}\right)}\right) & =\limsup _{\sigma \rightarrow 0} \sigma^{-s_{0}} \hat{\mathcal{H}}^{s_{0}}\left(\mathcal{S}_{j}(u) \cap \overline{B_{\frac{\sigma}{2}}\left(x_{0}\right)}\right) \\
& \geq 2^{-s_{0}} .
\end{aligned}
$$

Let $\left\{v_{i}\right\}$ and $v_{0}$ be blow up maps and tangent map of $v$ at $x_{0}$. For $i=1,2, \ldots$, define

$$
S_{i}=\left\{x \in \overline{B_{\frac{1}{2}}(0)}: x \in \mathcal{S}_{j}\left(v_{i}\right)\right\}
$$


and

$$
S_{0}=\left\{x \in \overline{B_{\frac{1}{2}}(0)} \cap \mathcal{R}(u):\left|\nabla v_{0}\right|=0\right\} \cup \mathcal{S}\left(v_{0}\right) .
$$

By (65), we have

$$
\lim _{i \rightarrow \infty} \hat{\mathcal{H}}^{s_{0}}\left(S_{i}\right) \geq 2^{-s_{0}}
$$

We next claim that

$$
\text { if } x_{i} \in S_{i} \text { and } x_{i} \rightarrow x \text {, then } x \in S_{0} .
$$

Since $x_{i} \rightarrow x, g_{i} \rightarrow g(0)$ uniformly, we obtain by the trace theory (cf. Theorem $1.12 .2[\mathrm{KS} 1])$ that

$$
\int_{\partial B_{r}(x)} d^{2}\left(v_{0}, P_{0}\right) d s=\lim _{i \rightarrow \infty} \int_{\partial B_{r}\left(x_{i}\right)} d^{2}\left(v_{i}, P_{0}\right) d s .
$$

Let $\bar{x}_{i} \in \mathcal{S}_{j}(u)$ be the point which corresponds to $x_{i} \in \mathcal{S}_{j}\left(v_{i}\right) \cap B_{1}(0)$ in the construction of the the blow up map $v_{i}$. Then with $\alpha_{i}=\operatorname{Ord}^{v}\left(\bar{x}_{i}\right)$, we have by Corollary 31 and with $A$ as in Corollary 30

$$
\frac{I_{x_{i}}^{v_{i}}(r)}{r^{n+1+2 \epsilon_{0}}} \leq \frac{I_{x_{i}}^{v_{i}}(r)}{r^{n-1+2 \alpha_{i}}} \leq c \frac{I_{x_{i}}^{v_{i}}\left(r_{0}\right)}{r_{0}^{n-1+2 \alpha_{i}}} \leq c \frac{I_{x_{i}}^{v_{i}}\left(r_{0}\right)}{r_{0}^{n-1+2 A}}
$$

Taking the limit as $i \rightarrow \infty$, we obtain

$$
\frac{I_{x}^{v_{0}}(r)}{r^{n+1+2 \epsilon_{0}}} \leq c \frac{I_{x}^{v_{0}}\left(r_{0}\right)}{r_{0}^{n-1+2 A}}
$$

In order to prove (67) notice that if $x \in \mathcal{R}\left(v_{0}\right)$, then

$$
\omega_{n}\left|\nabla v_{0}\right|^{2}(x)=\lim _{r \rightarrow 0} \frac{I_{x}^{v_{0}}(r)}{r^{n+1}} \leq c \lim _{r \rightarrow 0} \frac{I_{x}^{v_{0}}\left(r_{0}\right)}{r_{0}^{n-1+2 A}} r^{2 \epsilon_{0}}=0
$$

and this proves (67).

We now claim that for any $r \in(0,1)$ and any $s>0$,

$$
\hat{\mathcal{H}}^{s}\left(S_{0}\right) \geq \limsup _{i \rightarrow 0} \hat{\mathcal{H}}^{s}\left(S_{i}\right) \text {. }
$$

To prove (68), note that for a given $\epsilon_{1}>0$, choose a covering $\left\{B_{r_{l}}\left(x_{l}\right)\right\}_{l=1}^{N}$ of $S_{0}$ such that

$$
\hat{\mathcal{H}}^{s}\left(S_{0}\right)+\epsilon_{1} \geq \sum_{l=1}^{N} r_{l}^{s}
$$


Note here that it is enough to consider finite coverings since $S_{0}$ is closed by Lemma 15 and hence compact. By $(67),\left\{B_{r_{i}}\left(x_{i}\right)\right\}_{i=1}^{N}$ is a covering of $S_{i}$ for $i$ sufficiently large. Hence, for $i$ sufficiently large,

$$
\hat{\mathcal{H}}^{s}\left(S_{0}\right)+\epsilon_{1} \geq \sum_{j=1}^{N} r_{j}^{s} \geq \hat{\mathcal{H}}^{s}\left(S_{i}\right)
$$

Since $\epsilon_{1}$ is arbitrary, this proves (68).

In particular, let $s=s_{0}>n-2$ in (68) and combine with (66) to obtain

$$
\hat{\mathcal{H}}^{s_{0}}\left(S_{0}\right) \geq \limsup _{i \rightarrow 0} \hat{\mathcal{H}}^{s_{0}}\left(S_{i}\right) \geq 2^{-s_{0}}
$$

By Theorem 6.4 [GS], we have that $\operatorname{dim}_{\mathcal{H}}\left(\mathcal{S}\left(v_{0}\right)\right) \leq n-2$. Furthermore, since $v_{0}$ maps a neighborhood of $x \in \mathcal{R}(u)$ into Euclidean space, $\operatorname{dim}_{\mathcal{H}}\{x \in \mathcal{R}(u)$ : $\left.\left|\nabla v_{0}\right|^{2}(x)=0\right\} \leq n-2$. Thus, $\operatorname{dim}_{\mathcal{H}}\left(S_{0}\right) \leq n-2$ which is a contradiction.

Theorems 2 and 3 which improve Propositions 27 and 34 and Corollary 4 of the introduction are now immediate consequences of the Theorem 1 .

\section{References}

[C] K. Corlette. Archimedean superrigidity and hyperbolic geometry. Ann. of Math. 135 (1992) 165-185.

[DM] G. Daskalopoulos and C. Mese. $\epsilon$-regularity theorem for harmonic maps into DM-complexes and buildings. To appear in Volume honoring Richard Schoen's 60th Birthday.

[DMV] G. Daskalopoulos, C. Mese and A. Vdovina. Superrigidity of hyperbolic buildings. Submitted for publication.

[Fe] H. Federer. Geometric Measure Theory. Die Grundlagen der math. Wissenschaften, Band 153, Springer-Verlag, Berlin and New York, 1969.

[GS] M. Gromov and R. Schoen. Harmonic maps into singular spaces and p-adic superrigidity for lattices in groups of rank one. Publ. Math. IHES 76 (1992) 165-246. 
[J] J. Jost. Nonpositive curvature: geometric and analytic aspects. Lectures in Mathematics. ETH Zürich, Birkhäuser Verlag 1997.

[JY] J. Jost and S.T Yau. Harmonic maps and superrigidity. Proc. of Symposia in Pure Math. Vol 54 (1993), Part 1.

[KS1] N. Korevaar and R. Schoen. Sobolev spaces and harmonic maps into metric space targets. Comm. Anal. Geom. 1 (1993) 561-659.

[KS2] N. Korevaar and R. Schoen. Global existence theorem for harmonic maps to non-locally compact spaces. Comm. Anal. Geom. 5 (1997) 333-387.

[Me] C. Mese. Harmonic maps into spaces with an upper curvature bound in the sense of Alexandrov. Math. Z. 242 (2002) 633-661.

[MSiY] N. Mok, Y.T. Siu and S.K. Yang. Geometric superrigidity. Invent. Math. 113 (1993) 57-83.

[Se] T. Serbinowski. Boundary regularity of harmonic maps to nonpositively curved metric spaces. Comm. Anal. Geom. 2 (1994) 139-154.

[Si] Y.T. Siu. The complex analyticity of harmonic maps and the strong rigidity of compact Kähler manifolds. Ann. of Math. 112 (1980) 73111. 